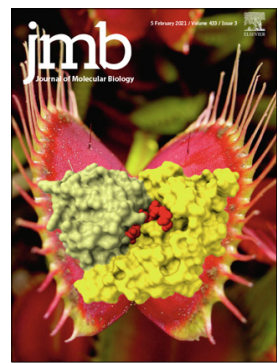

\title{
Discriminative SKP2 Interactions with CDK-Cyclin Complexes Support a Cyclin A-Specific Role in p27KIP1 Degradation
}

\author{
Marco Salamina ${ }^{1}$, Bailey C. Montefiore ${ }^{1}$, Mengxi Liu ${ }^{2}$, Daniel J. Wood ${ }^{1}$, \\ Richard Heath ${ }^{1}$, James R. Ault ${ }^{3}$, Lan-Zhen Wang ${ }^{1}$, Svitlana Korolchuk ${ }^{1}$, \\ Arnaud Baslé ${ }^{4}$, Martyna W. Pastok ${ }^{1}$, Judith Reeks ${ }^{1}$, Natalie J. Tatum ${ }^{1}$, \\ Frank Sobott $^{3}$, Stefan T. Arold ${ }^{5,6}$, Michele Pagano ${ }^{2}$, Martin E. M. Noble ${ }^{1 *}$ and \\ Jane A. Endicott ${ }^{1 *}$
}

1 - Newcastle University Centre for Cancer, Translational and Clinical Research Institute, Newcastle University, Paul O'Gorman Building, Framlington Place, Newcastle upon Tyne NE2 4HH, UK

2 - Department of Biochemistry and Molecular Pharmacology, Perlmutter NYU Cancer Center, New York University Grossman School of Medicine, and Howard Hughes Medical Institute, The Alexandria Center of Life Science, East Tower, 450 E, 29th Street, New York, NY 10016, USA

3 - Astbury Centre for Structural Molecular Biology, School of Molecular and Cellular Biology, University of Leeds, Leeds LS2 9JT, UK

4 - Biosciences Institute, Newcastle University, Framlington Place, Newcastle upon Tyne NE2 4HH, UK

5 - Division of Biological and Environmental Sciences and Engineering (BESE), Computational Bioscience Research Center (CBRC), King Abdullah University of Science and Technology (KAUST), Thuwal 23955-6900, Saudi Arabia

6 - Centre de Biochimie Structurale, CNRS, INSERM, Université de Montpellier, 34090 Montpellier, France

Correspondence to Martin E.M. Noble and Jane A. Endicott: jane.endicott@ncl.ac.uk (J.A. Endicott) https://doi.org/10.1016/j.jmb.2020.166795

Edited by Richard W. Kriwacki

\begin{abstract}
The SCF ${ }^{\text {SKP2 }}$ ubiquitin ligase relieves $\mathrm{G} 1$ checkpoint control of CDK-cyclin complexes by promoting p27KIP1 degradation. We describe reconstitution of stable complexes containing SKP1-SKP2 and CDK1-cyclin B or CDK2-cyclin A/E, mediated by the CDK regulatory subunit CKS1. We further show that a direct interaction between a SKP2 N-terminal motif and cyclin A can stabilize SKP1-SKP2-CDK2-cyclin A complexes in the absence of CKS1. We identify the SKP2 binding site on cyclin A and demonstrate the site is not present in cyclin $\mathrm{B}$ or cyclin $\mathrm{E}$. This site is distinct from but overlapping with features that mediate binding of $\mathrm{p} 27 \mathrm{KIP} 1$ and other G1 cyclin regulators to cyclin A. We propose that the capacity of SKP2 to engage with CDK2-cyclin A by more than one structural mechanism provides a way to fine tune the degradation of p27KIP1 and distinguishes cyclin A from other G1 cyclins to ensure orderly cell cycle progression.

(c) 2021 The Author(s). Published by Elsevier Ltd. This is an open access article under the CC BY license (http://creativecommons.org/licenses/by/4.0/).
\end{abstract}

\section{Introduction}

SKP2 (FBXL1) and SKP1 (S-phase kinaseassociated proteins 1 and 2) were identified as components of a pentameric cyclin-dependent kinase 2 (CDK2)-cyclin A-cyclin-dependent kinases regulatory subunit 1 (CKS1)-containing complex whose levels were found to be elevated in transformed cell lines compared to their nontumorigenic counterparts. ${ }^{1}$ SKP2 has since been characterized as a member of a large protein family that shares the F-box motif that binds to SKP1 $1^{2,3}$ 
and selectively recruits phosphorylated substrates into SCF (SKP1-Cullin1/Cdc53-F-box protein) E3 ubiquitin ligase complexes. These complexes polyubiquitinate their substrates targeting them for degradation by the proteasome,$^{4-7}$ reviewed in. ${ }^{8-10}$

The central element of the SKP2 structure (residues 94-140) comprises the F-box that is sandwiched between a larger C-terminal leucinerich repeat (LRR) domain, and a smaller $\mathrm{N}$ terminal sequence that is predicted to be unstructured. The SKP2 LRR domain and tail recognize phosphorylated substrates that include cyclin-dependent kinase inhibitors (CKIs, including p27KIP1 (p27)), retinoblastoma-like protein 2 and the transcription factor FOXO1 (reviewed in $11,12)$. Unusually, SKP2 cooperates with an accessory protein, CKS1, to recognize the phosphorylated T187 residue of p27 that signals its degradation. ${ }^{13-17}$ The unstructured N-terminal sequence contains a functional $\mathrm{D}$-box near the $\mathrm{N}$ terminus that is recognized by Cdh1, ${ }^{18,19}$ as well as sequences that interact with cyclin $A^{20}$ and pRB. ${ }^{21}$ It is also site-specifically phosphorylated (for example by CDK2-cyclin A at S64) ${ }^{22}$, and acetylated, suggesting mechanisms by which protein association might be regulated. ${ }^{16,22-28}$

The cyclin A binding site on SKP2 has been mapped to four residues within the $\mathrm{N}$-terminal portion of SKP2. ${ }^{20}$ The character and relative disposition of these residues (L32, L33, S39 and L41 (UniProt entry Q13309 isoform 1 numbering)) suggests a hydrophobic docking sequence that binds to cyclin A. The SKP2 binding site on cyclin A has been proposed to lie within the cyclin $A$ unstructured sequence $\mathrm{N}$-terminal to the two tandem cyclin box folds. ${ }^{20}$ However, SKP2 and p27 association with CDK2-cyclin A is mutually exclusive suggesting that the SKP2 binding site must overlap with that of p27 elsewhere on the cyclin A structure. ${ }^{20}$

Cyclin A is required for DNA replication and, in Sphase, associates with and activates CDK2. ${ }^{29,30}$ Cyclin $E$ is an alternative activating partner of CDK2 that is predominantly expressed in late G1 phase. CKIs of the CIP/KIP class (of which p27 is a member) and selected CDK substrates share a consensus RXL sequence (single letter amino acid code in which $X$ denotes any amino acid) that makes a significant contribution to their binding to CDK-cyclin complexes. ${ }^{31}$ This motif binds to the cyclin recruitment site, a hydrophobic patch on the surface of the $\mathrm{N}$-terminal cyclin box fold (N-CBF) that is conserved in cyclins $A, B, D$ and $E .^{32,33}$ The crystal structure of a CDK2-cyclin A-p27 complex revealed that an $\mathrm{N}$-terminal $\mathrm{p} 27$ fragment both drapes across the surface of cyclin $A$ and integrates itself into the fold of the N-lobe of CDK2. ${ }^{32}$ p27 is an intrinsically disordered protein and biophysical characterization of this interaction suggested an induced fit model in which three distinct structural units can be distinguished in the p27 N-terminal sequence. ${ }^{34,35}$ The most $\mathrm{N}$ - and C-terminal of these units are unstructured in solution and bind to the cyclin A recruitment site and CDK2 $\mathrm{N}$-lobe respectively. The central unit is $\alpha$-helical and docks onto the surface of cyclin A. RXL-containing CDK substrates are proposed to have more limited interactions with the surface of the CDK-cyclin, docking at the cyclin A recruitment site and thereby effectively increasing local concentration, which enhances substrate engagement within the CDK2 catalytic cleft. ${ }^{36,37}$ Apart from their interactions with CDK partners, p27, and short motifs from RXLcontaining substrates, no other protein interaction sites have been structurally characterized on the surface of cyclins A, B, D or E that control the cell cycle. In S. cerevisiae a conserved "LP" docking site that binds a consensus LXF motif has been identified in Cln1/2 and Ccn1 cyclins adjacent to the recruitment site. ${ }^{38}$

In this study we identify the features of cyclin A that distinguish it from cyclin $\mathrm{E}$ and cyclin $\mathrm{B}$ and that mediate formation of a SKP1-SKP2-CDK2cyclin A-CKS1 pentameric complex. Using purified proteins we show that a stable CDK2-cyclin ASKP1-SKP2-CKS1 complex can be formed either through CKS1 bridging CDK2 and SKP2 ${ }^{16}$ or through a direct interaction between the SKP2 Nterminal sequence and a cyclin A SKP2 binding site that we identify in this study. We show that the cyclin A SKP2 and p27 binding sites have unique and overlapping elements and that SKP2 and p27 binding to cyclin A is mutually exclusive. We propose a model in which recruitment of non-phosphorylated p27 to CDK1/2-cyclin A/B/E-SKP1-SKP2-CKS1 complexes is mediated by CKS1. Subsequent phosphorylation of p27 is affected by the SKP2 Nterminal cyclin $A$ binding motif that recruits a catalytic CDK-cyclin A to form an octameric complex that affects phosphorylation of p27 in cis. This SKP2-cyclin A interaction imparts a unique activity to cyclin A to promote p27 degradation that distinguishes it from cyclins $E$ and $B$. We hypothesize that the interactions between CDK1 or CDK2 with CKS1, and the interaction between SKP2 and cyclin A act together to determine the efficiency of p27 T187 phosphorylation and its subsequent SCF ${ }^{\mathrm{SKP} 2}$. dependent degradation.

\section{Results}

\section{The cyclin A N-terminal cyclin box fold has a SKP2 binding site}

To identify the SKP2 binding site on cyclin A we exploited the knowledge that (i) SKP2 cannot form a pentameric CDK2-cyclin-SKP1-SKP2-CKS1 complex if cyclin $E$ replaces cyclin $A^{1}$; (ii) residues in the SKP2 N-terminal sequence prior to the start of the F-box are required for SKP2 to bind to cyclin $\mathrm{A}^{20,39}$ and (iii) SKP2 and p27 binding to cyclin A are mutually exclusive. ${ }^{20}$ We first confirmed in a reconstituted system using purified proteins that in the absence of CKS1, cyclin A but not cyclin E can form 
a complex with CDK2, SKP1 and SKP2 (Figure 1 (a)) and cyclin A but not CDK2 binds directly to SKP1-SKP2 (Supplementary Figure S1(A)). We next constructed a co-expression system to pro- duce a SKP1-SKP2 complex comprising SKP1 and the first 140 residues of SKP2 (SKP1SKP2N), encoding the $\mathrm{N}$-terminal regulatory sequence (residues 1-93) and the F-box, (residues

(a)

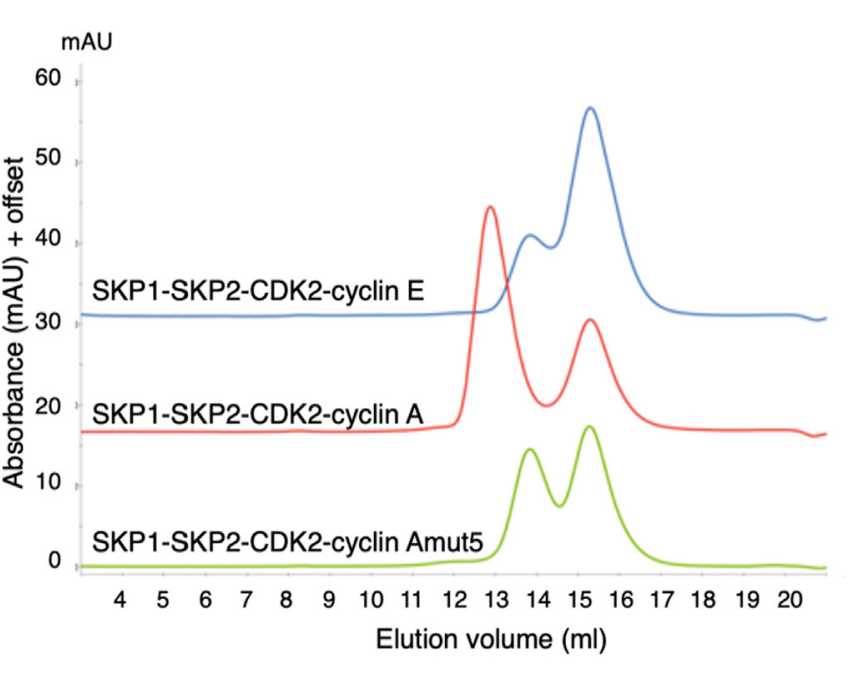

(b)
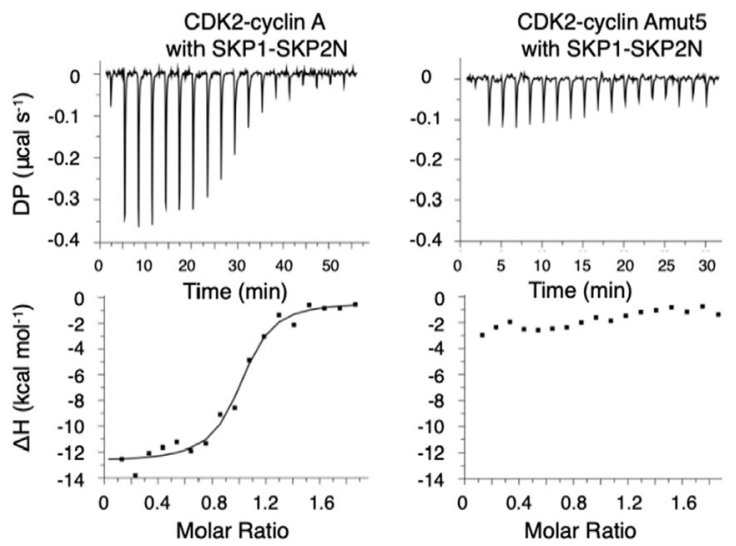

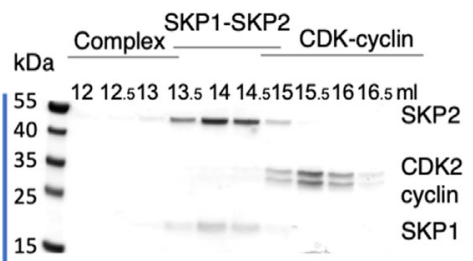

SKP1-SKP2-CDK2-cyclin E

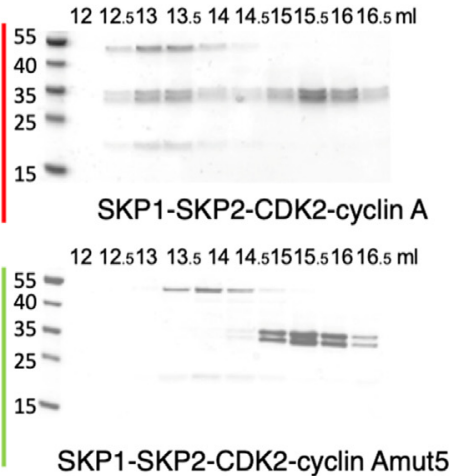

(c)

Figure 1. Identification of a SKP2 binding site on cyclin A. (a) SKP1-SKP2 forms a stable complex with CDK2-cyclin A (red (middle) chromatogram), but not with CDK2-cyclin E (blue chromatogram) or CDK2-cyclin Amut5 (green (lower) chromatogram). Recombinant SKP1-SKP2, CDK2-cyclin A (cyclin A residues 174-432, 33) and CDK2-cyclin E complexes (cyclin E residues 96-378, 58) were purified separately. Chromatograms are to the same scale but have been offset on the y-axis to aid comparison. Accompanying SDS-PAGE analysis of selected size-exclusion chromatography fractions is shown on the right. Samples were visualized by Instant Blue staining. The molecular weights of SKP2, CDK2, cyclin A/E C-terminal constructs and SKP1 are respectively circa $48 \mathrm{kDa}, 34 \mathrm{kDa}, 30 / 33 \mathrm{kDa}$ and $19 \mathrm{kDa}$ respectively. Chromatogram is representative of two replicates carried out using independently prepared proteins. (b) Isothermal titration calorimetry (ITC) thermograms to assess SKP1-SKP2N binding to CDK2-cyclin A and CDK2-cyclin A mutants. SKP1-SKP2N binds to CDK2-cyclin A (LHS panel) but shows no detectable binding to CDK2cyclin Amut5 (RHS panel). ITC thermograms shown are one of two replicates carried out using independently prepared proteins. (c) Characterization of cyclin A mutants to identify the cyclin A SKP2 binding site. Location of the cyclin Amut5 mutation. Cyclin A residues M246 and S247 are highlighted in red in the context of the CDK2-cyclin Ap27 structure (PDB entry 1JSU). CDK2 and p27 are drawn in ribbon representation (green and magenta respectively) and the surface of cyclin A is rendered in blue. W217 that is at the center of the cyclin A recruitment site that binds the RXL motif is highlighted in magenta. Data analysis is described in Table 1. 
94-140). Using isothermal titration calorimetry (ITC) we confirmed that SKP1-SKP2N binds to CDK2-cyclin A (with an affinity of $298 \pm 17 \mathrm{nM}$ (Figure 1(b)) but does not bind to CDK2 alone (Table 1 and Supplementary Figure S1(B)). Mapping the sequence conservation between E-cyclins and Acyclins onto the structure of cyclin A revealed several overlapping surface patches that are highly conserved within but not between the cyclin $A$ and $E$ families. A subset of these sites that are close to, but do not form part of, the cyclin A binding site for p27 were selected for further study (Table 1, Figure 1 and Supplementary Figure S2(A)).

To test these potential SKP2 binding sites we again used ITC to compare the binding of SKP1SKP2N to the authentic cyclin A and cyclin A mutant proteins (Table 1 and Supplementary Figure S2(B)). Where all the selected sequence loci in cyclin $A$ were mutated to replicate the sequence of cyclin $\mathrm{E}$ (generating a variant termed cyclin Aglobal), binding to SKP1-SKP2N was completely abrogated. Individually mutating certain subsets of these sequence loci (284-289 to generate cyclin Amut2, 290-299 to generate cyclin Amut3 or 299305 to generate cyclin Amut6) had no discernible or relatively little impact on SKP2-cyclin A association. By contrast, changing the cyclin A sequence at residues 244-247 (SSMS, Supplementary Figure S2(A), (B)) or at 246-247 (MS, Figure 1(b) and (c)) to the equivalent cyclin $\mathrm{E}$ sequences (ATQEN or QEN respectively, generating the respective variants cyclin Amut7 and cyclin Amut5 had a profound effect on the interaction. To verify the ITC results we used SEC to confirm that full-length SKP2-SKP1 does not form a complex with CDK2-cyclin Amut5 (Figure 1 (a)). Finally, to confirm the structural and functional integrity of cyclin Amut5 we prepared the CDK2cyclin Amut5 complex and both determined its crystal structure (Supplementary Figure S2(C), Supplementary Table S1, PDB entry 6SG4) and compared its activity with CDK2-cyclin A towards a model RXL-containing peptide substrate derived from $\mathrm{p} 107^{40}$ (Supplementary Figure S2(D) and Supplementary Table S2). The structure shows that the insertion has not affected the overall fold and the $k_{\text {cat }} /$ $\mathrm{K}_{\mathrm{m}}$ values for each complex towards this substrate were comparable.

\section{Cyclin A has distinct but overlapping SKP2 and $\mathbf{p 2 7}$ binding sites}

This site which we have termed the "SSMS site", identified by cyclin Amut5, represents a novel site of cyclin A protein interaction. It is distant from the cyclin recruitment site but is close to residues that interact with p27 (Figure 1(c)). Given the locations of the SSMS site, the RXL recruitment site and the extended p27 $\alpha$-helical cyclin A binding sequence we hypothesized that the extended SKP2 binding site might overlap with either the p27 RXL motif or $\alpha$-helical cyclin A binding sites.
(Respectively also referred to as p27 sequence "D1" (residues 27-37) and "linker helix" (residues 38-58). ${ }^{41}$

To test this model, we first confirmed using surface plasmon resonance (SPR) and ITC that the binding of cyclin A to p27 was not affected by the mut5 mutation (Figure 2(a) and (b), Table 1). GST-p271-106 (p27M) was immobilized on the SPR chip surface and CDK2-cyclin A and CDK2cyclin Amut5 were flowed over as analytes. The $\mathrm{K}_{\mathrm{d}}$ values determined by this method were not significantly different, being respectively $15.50 \pm 0$. $88 \mathrm{nM}$ and $25.00 \pm 0.82 \mathrm{nM}(\mathrm{p}>0.05)$, and similar to values previously reported. ${ }^{34}$

We next prepared a series of cyclin A variants, mutated at residues that interact with p27 or are located close to the recruitment or SSMS sites, and tested their ability to bind to p27 and to SKP1SKP2 using ITC (Figure 2(c), Supplementary Figure $S 3(A)$ and Table 1). For this assay we again used the SKP1-SKP2N construct and, in order to assess only the cyclin A binding properties of $\mathrm{p} 27$, we used a truncated fragment of p27 encompassing residues 23 to 51 (p27MS) that includes the RXL motif and does not contact CDK2. Measured under the ITC conditions, p27MS binds to CDK2-cyclin A with an affinity of $350 \pm 36 \mathrm{nM}$ (Figure 2(b) and Table 1). Variants of cyclin A were prepared to probe the contribution to protein binding made by the recruitment site (variant cyclin A_W217K), by an adjacent hydrophobic pocket that binds to F33 of p27 (variant cyclin A_1213D), by a residue from helix $\alpha 5$ that does not directly contact p27 (variant cyclin A_M210D), and by the cyclin A surface groove that binds the p27 linker helix (variants cyclin A_R293E and cyclin A_L297K). Cyclin A_R293E was engineered because R293 interacts with p27 E46, whereas cyclin A_L297K was chosen because L297 contributes to a hydrophobic pocket that accepts p27 residues L45 and C49 and the comparable residue is a lysine in cyclin B (32, Figure 2(c)).

As expected, cyclin Amut5 bound tightly to p27 (Figure 2(b), Table 1) but cyclin A mutants W217K, I213D, R293E (Figure 2(c) and Table 1) and L297K (Supplementary Figure S3(A) and Table 1) showed reduced binding to p27 (significance calculated at $p<0.05$ ). The introduction of the W217K mutation also disrupted binding to SKP1-SKP2N (Figure 2(c)) suggesting that SKP2 binding also depends on the cyclin partner having an intact recruitment site, despite SKP2 not containing a canonical RXL motif. We confirmed W217K mutation does not affect the overall integrity of the cyclin A fold by differential scanning fluorimetry (Supplementary Figure S3 (B)). CDK2-cyclin A, CDK2-cyclin Amut5 and CDK2-cyclin AW217K have comparable $\mathrm{T}_{\mathrm{m}}$ mean values of $48.75( \pm 0.22){ }^{\circ} \mathrm{C}, 47.05( \pm 0.23){ }^{\circ} \mathrm{C}$ and $47.68( \pm 0.42){ }^{\circ} \mathrm{C}$ respectively. 
Mutant M210D at the start of the MRAIL sequence had little effect on the binding of either p27 or SKP2 (Supplementary Figure S3(A)). This residue does not contact p27 in the CDK2-cyclin A-p27 structure and this result suggests that it lies distant from the SKP2 binding site as well. Intriguingly, introduction of the I213D mutation reduced affinity for p27 circa 5-fold, but enhanced affinity for SKP2 circa 5-fold (Figure 2(C) and Table 1). Cyclin A mutations in $\alpha 5$ also distinguish p27 and SKP2 binding, suggesting that p27 and SKP2 do not share common interactions with this region. Binding of SKP2 was severely compromised by the introduction of the L297K mutation into cyclin A, but we did not measure a significant effect for the R293E mutation that ablates p27MS binding ( $p>0.05)$.

We next characterised CDK2-cyclin A, CDK2cyclin A-p27 and CDK2-cyclin A-SKP2-SKP2 complexes by hydrogen-deuterium exchange mass spectrometry (HDX-MS) to distinguish p27 and SKP2 binding to cyclin A by an orthogonal technique, and to explore the extent of the cyclin A surface affected by SKP2 binding (Figure 3, Supplementary Figure S4). For these experiments, p27M (residues 1-106) and $\triangle 20 S K P 2 N$ (residues 20-140) constructs were employed. We determined the comparative uptake profiles for CDK-cyclin A on its own, or in complex with either p27M or SKP1- 20 SKP2N. Determination of deuterium uptake was made at the peptide level (following online pepsin digestion of the proteins) for deuterium incubation times of
$30 \mathrm{~s}, 1 \mathrm{~min}, 2 \mathrm{~min}, 10 \mathrm{~min}$ and $60 \mathrm{~min}$. Following data processing, differential exchange between the conditions was identified using PAVED software ${ }^{42}$ to determine the highlighted statistically significant differences in deuterium uptake (Figure 3, Supplementary Figure S4). The significant areas of modulated exchange on cyclin $A$ are mainly focussed around five regions however the exchange 'fingerprint' is unique in either case. In the presence of p27M four areas show increased protection (Figure 3(a), yellow, lilac, purple and pink) corresponding to positions 251-255, 270278, 280-296 and 302-314 respectively. As expected, these sequences map closely to the p27 binding site (PDB 1JSU) and to residues R293 and L297 mutation of which affects p27 binding (Table 1). In contrast in the CDK2-cyclin ASKP1- $\triangle 20 S K P 2 N$ complex, cyclin A peptides show significant protection at positions 201-210 (red), 248-255 (yellow and includes the SSMS sequence), and to a smaller extent 280-287 (purple) (Figure 3(b)). Mutation of cyclin A residues 1213, W217 and L297 also affect p27 and SKP2 binding to cyclin $A$ (Table 1). However, fragments 213-218 and 295-299 that respectively include these residues were already highly protected even in the absence of p27M and $\triangle 20$ SKP2N SKP2 (Supplementary Figure S4) and did not undergo significant exchange in either the CDK2-cyclin A-p27 or CDK2-cyclin A- $\triangle 20$ SKP2N complexes over the time course of the experiment.

Taken together, the results of the ITC and HDXMS experiments suggest a model in which p27 and

Table 1 Cyclin A mutants display a range of binding affinities to SKP1-SKP2 and p27. $\mathrm{K}_{\mathrm{d}}$ values were determined by isothermal titration calorimetry (ITC).

\begin{tabular}{|c|c|c|c|c|}
\hline Cyclin A & $\begin{array}{l}\text { Residues } \\
\text { mutated }\end{array}$ & Residue changes & $\begin{array}{l}\text { SKP1- } \\
\text { SKP2N } \\
\mathrm{K}_{\mathrm{d}} \pm(\mathrm{nM})\end{array}$ & $\begin{array}{l}\mathrm{p} 27 \mathrm{MS} \\
\mathrm{K}_{\mathrm{d}} \pm(\mathrm{nM})\end{array}$ \\
\hline${ }^{1}$ Wild-type & none & none & $298 \pm 17^{5}$ & $350 \pm 36^{5}$ \\
\hline${ }^{1}$ mut2 & $284-289$ & DTYTKK to GACSGD & $340 \pm 60^{6}$ & $-{ }^{3}$ \\
\hline${ }^{1}$ mut3 & 290-299 & QVLRMEHLVL to EILTMELMIM & $658 \pm 101^{6}$ & - \\
\hline${ }^{1}$ mut5 & $246-247$ & MS to QEN & $\mathrm{ND}^{4,5}$ & $176 \pm 1.5^{5}$ \\
\hline${ }^{1}$ mut6 & 299-305 & LKVLTFD to MKALKWR & $1690 \pm 340^{6}$ & - \\
\hline${ }^{1}$ mut7 & $244-247$ & SSM-S to ATQEN & $\mathrm{ND}^{6}$ & - \\
\hline${ }^{1}$ global & $\begin{array}{l}244-247 \\
284-305\end{array}$ & $\begin{array}{l}\text { S244A, S245T, M246Q, S247E+N, D284G, T285A, Y286C, T287S, } \\
\text { K288G, K289D, Q290E, V291I, R293T, H296L, L297M, V298I, } \\
\text { L299M, V301A, T303K, F304W, D305R }\end{array}$ & $\mathrm{ND}^{6}$ & - \\
\hline${ }^{2} W 217$ & 217 & W217K & $\mathrm{ND}^{5}$ & $\mathrm{ND}^{5}$ \\
\hline${ }^{2} \mathrm{M} 210$ & 210 & M210D & $894 \pm 120^{6}$ & $401 \pm 177^{6}$ \\
\hline${ }^{2} 1213$ & 213 & I213D & $50.7 \pm 11.0^{5}$ & $1800 \pm 160^{5}$ \\
\hline${ }^{2} \mathrm{R} 293$ & 293 & R293E & $728 \pm 282^{5}$ & $\mathrm{ND}^{5}$ \\
\hline${ }^{2} \mathrm{~L} 297$ & 297 & L297K & $\mathrm{ND}^{5}$ & $\mathrm{ND}^{5}$ \\
\hline
\end{tabular}


(a)

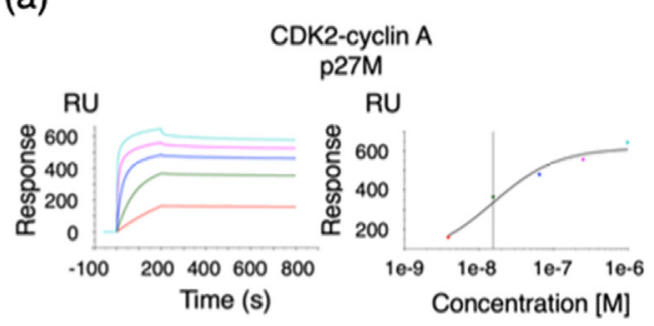

(b)

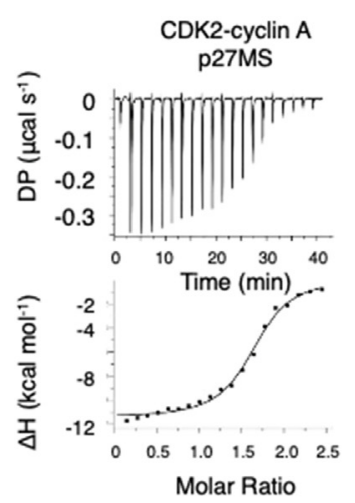

(c)
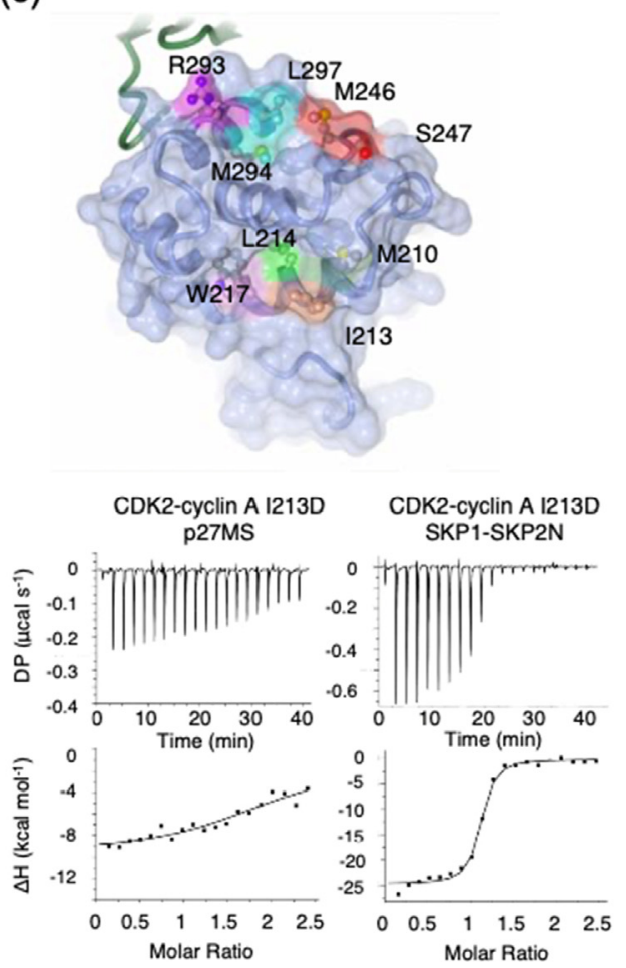
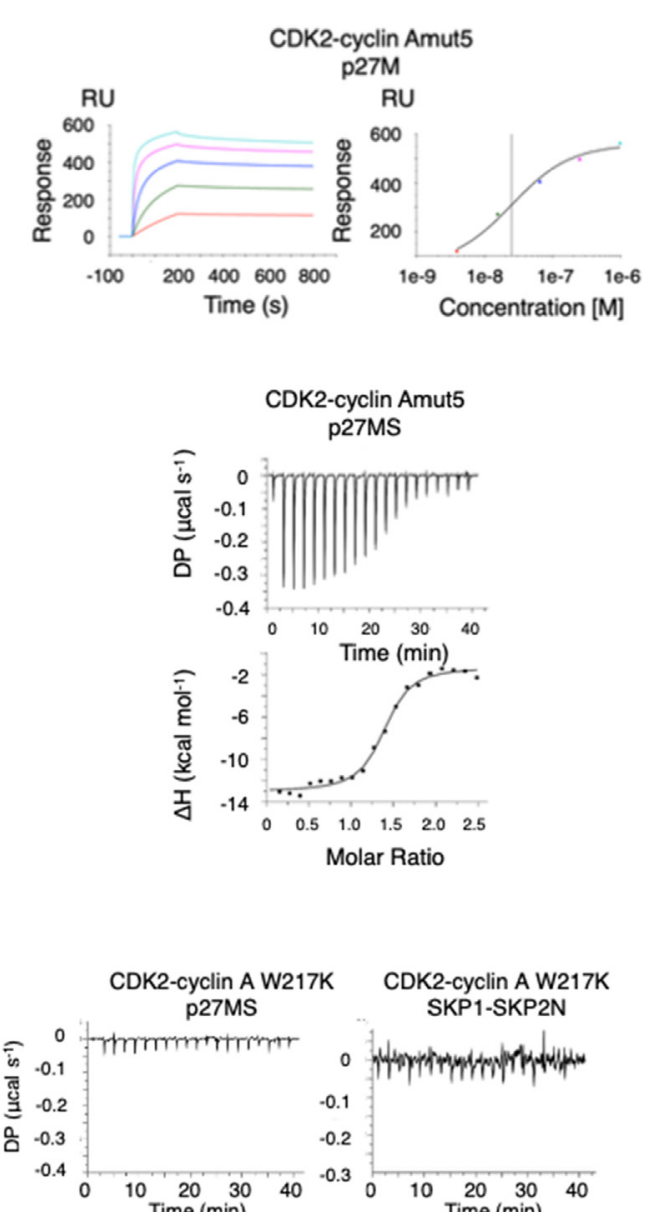

CDK2-cyclin A W217K
SKP1-SKP2N
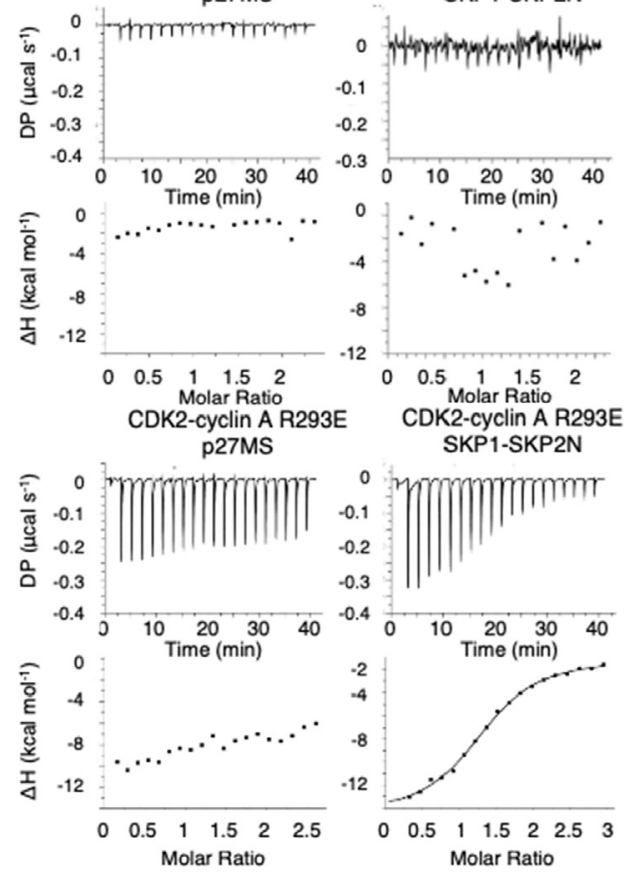

Figure 2. Defining the SKP2 binding site on cyclin A. (a) Surface plasmon resonance to measure the interaction between p27 and CDK2-cyclin A and CDK2-cyclin Amut5. GST-p27M (residues 1-106) was bound to a CM5 sensor-chip functionalized with anti-GST antibody and the CDK2-cyclin A complexes were flowed over at increasing concentrations. $\mathrm{K}_{\mathrm{d}}$ values were derived using Biacore S200 evaluation software. SPR sensorgrams are representative of two repeats using independently prepared protein samples. (b) Isothermal titration calorimetry (ITC) to measure the interaction between p27MS (residues 23-51) and CDK2-cyclin A complexes. $K_{d}$ values and reaction conditions are tabulated in Table 1. (c) Cyclin A mutants distinguish SKP2 and p27 binding sites on cyclin A and reveal their shared and divergent binding elements. ITC thermograms for the binding of p27MS (residues 23-51) and SKP1-SKP2N to cyclin A_W217K, cyclin A_I213D (within the cyclin A a1 MRAIL sequence), cyclin A_R293E (on cyclin a5). ITC thermograms for other mutants discussed in the text are included in Figure S3(A). ITC thermograms shown are of one of two replicates carried out using independently prepared proteins. Data analysis is described in Table 1. 
SKP2 engage with the cyclin A RXL site and make related but distinct contacts with the $\mathrm{N}$-terminal end of the MRAIL motif. However, their paths subsequently diverge: whereas p27 interacts with the start of $\alpha 5$ to traverse the "top" of the cyclin A fold, SKP2 does not. Both protein binding sites then converge again at the $\mathrm{C}$-terminal end of cyclin $\mathrm{A} \alpha 5$ as defined by their shared sensitivity to the mutation L297K (Figure 2(c)).

\section{An extended SKP2 sequence binds to cyclin A}

Previous work has shown that SKP2 residues L32, L33, S39 and L41 interact with cyclin A. ${ }^{20}$ To confirm the importance of these residues and assess the extent of the SKP2 sequence that binds to cyclin A we prepared a series of authentic and mutant SKP1-SKP2N constructs and short SKP2 peptides and assessed their binding to CDK2cyclin A by ITC (Table 2, Supplementary Figure S5). Compared to binding of the authentic sequence, truncating SKP2N to start at F20 had little effect on the affinity but truncating to start at S30 significantly affected the SKP2N-cyclin A interaction (Supplementary Figure S5(A) and (B)). SKP1SKP2N_4A (with mutations L32A, L33A, S39A and $L 41 A$ ) showed circa 15 -fold decrease in binding to CDK2-cyclin A suggesting that other SKP2 residues contribute ((Supplementary Figure S5(C)) and compare with Figure 1(b)). Although the SKP2 N-terminal sequence contains a number of residues that are highly conserved, two tryptophan residues at positions W22 and W24 are prominent. Binding of SKP2 to cyclin A was not detectable when these residues were also mutated to alanine to generate SKP1-SKP2N_6A (mutations SKP2_4A and W22A and W24A) (Supplementary Figure S5(D)). Thus, the SKP2 sequence between residues F20 and E45 contributes substantially to cyclin A binding and we propose that this sequence constitutes a SKP2 cyclin A interacting motif (CAIM).

While an intact CAIM is required for SKP2 to bind to cyclin A, we were not able to detect stable binding of isolated CAIM peptides to cyclin A using ITC (Supplementary Figure $S 5(E)-(G)$ ), suggesting that the interaction is dependent on the context in which the CAIM is presented. To characterize this context-dependent interaction we analyzed a solution of CDK2-cyclin A and SKP1-SKP2N by small angle X-ray scattering (SAXS) (Figure 4, Supplementary Table S3). This analysis suggests a stable, fairly compact and rigid assembly. Within this assembly, we have modelled the SKP2 CAIM as a linear epitope that tethers the SKP2 to cyclin A. However, as we were not able to detect an interaction between the CAIM-containing peptides and cyclin A, we cannot distinguish between models in which the interaction is dependent on the context in which the CAIM is presented or is stabilized to bind by additional interactions within the assembly thereby rigidifying the resulting SKP1-SKP2N-CDK2-cyclin A complex.

\section{SKP2 binds to CDK2-cyclin A-CKS1 through two distinct interactions}

A tight (nM) interaction between CKS1 and the Cterminal sequence of SKP2 helps stabilize the pentameric CDK2-cyclin A-CKS1-SKP1-SKP2 complex. ${ }^{16}$ To assess the relative contributions of the CKS1-SKP2 and cyclin A-SKP2 interactions to the stability of the CDK2-cyclin A-CKS1-SKP1SKP2 pentameric complex, two sets of mutations were made to block each interaction and the resulting proteins were analyzed by SEC. To compromise the binding of SKP2 to CKS1, a SKP2 variant was constructed in which $\mathrm{F} 393$ was mutated to a glycine-serine pair (SKP2_C2, Supplementary Figure S6(A)), while cyclin Amut 5 was used to compromise the binding of cyclin A to the CAIM. By SEC, a stable pentameric complex forms when either the SKP2-cyclin A or CKS1-SKP2 interaction is intact, but in the absence of both sites the proteins migrate as CDK2-cyclin A-CKS1 and SKP1-SKP2 sub-complexes (Figure 5(a)). This analysis demonstrates that either site is sufficient to stabilize the pentameric complex in cell free systems. Incubating SKP1-SKP2 with an excess of CDK2-cyclin ACKS1 did not lead to the formation of a larger complex indicating that a (CDK2-cyclin A-CKS1) $2^{-}$ SKP1-SKP2 complex is not abundant under these conditions (Figure 5(a), magenta trace). However, we cannot exclude that such a complex might form dependent on effective local concentrations of CDK2-cyclin A. Using SKP1-SKP2, CDK2-cyclin E-CKS1 and CDK1-cyclin B-CKS1 we could also demonstrate that the CDK2-CKS1 or CDK1-CKS1 interface is sufficient to maintain a stable pentameric complex in the absence of a cyclin ASKP2 interaction (Supplementary Figure S6(B)). These results demonstrate that in the absence of CKS1, only CDK-cyclin A (and not -cyclin B or cyclin E) can form a stable complex with SKP1SKP2. However, if CKS1 is present, then it can bind to either CDK1 or CDK2 and SKP2 to "glue" the CDK-cyclin and SKP1-SKP2 binary complexes together. Furthermore, SEC analysis shows that p27-CDK2-cyclin A forms a stable complex with SKP1-SKP2 only in the presence of CKS1 (Supplementary Figure $5(C))$. The interaction between SKP2 and CKS1 is required to maintain the integrity of the hexameric CDK2-cyclin A-CKS1-SKP1SKP2-p27 complex, as p27 competes with SKP2 to displace the SKP2 CAIM from cyclin A. Taken together these observations suggest a model in which the recruitment of p27 into a complex with SKP1 and SKP2 is dependent on the presence of CKS1 and is independent of the identity of its CDK-cyclin partners. 
(a)
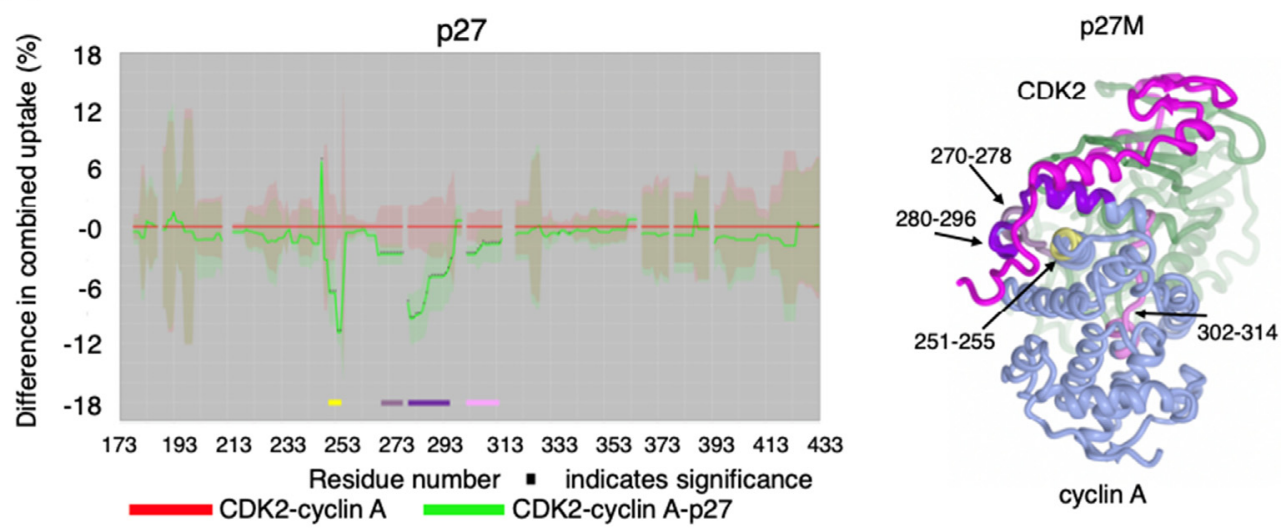

(b)
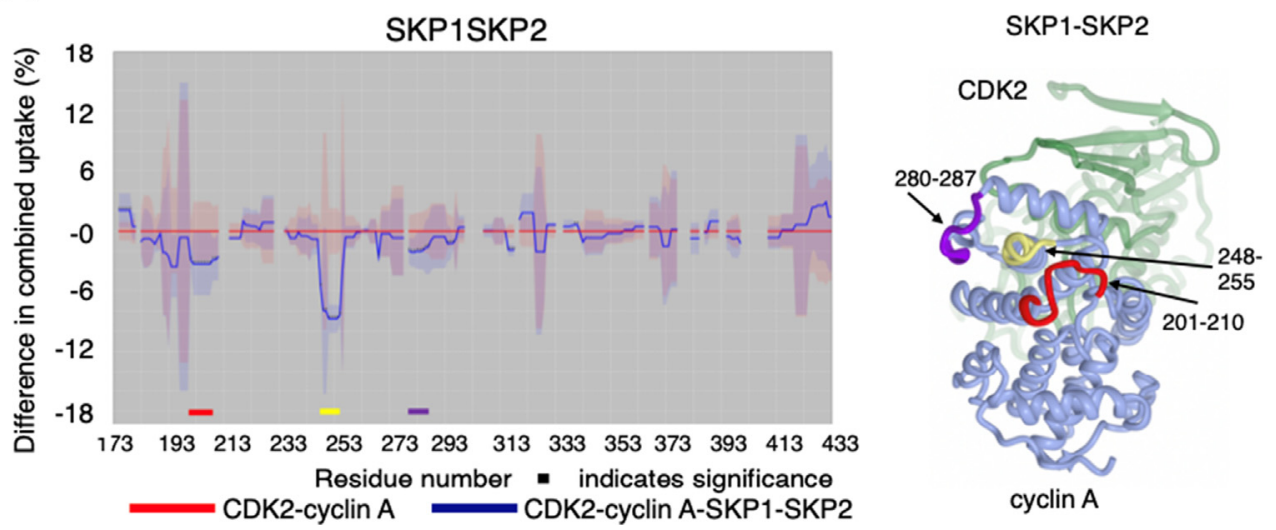

Figure 3. Hydrogen-deuterium exchange mass spectrometry distinguishes p27 and SKP2 binding to cyclin A. Differences in the combined relative fractional uptake of CDK2-cyclin A alone and in the presence of p27M (residues 1-106) (a) or SKP1- 20 SKP2N (SKP2 residues 20-140) (b) is visualised using the PAVED algorithm ${ }^{42}$ and plotted $^{2}$ against cyclin A residue number. Uptake plots shown were determined after 2.0 min deuterium incubation. Significant differences are marked by asterisks and the colored bars highlight areas discussed in the text. Peptides that show differences in fractional deuterium uptake when bound to either p27 or SKP1-SKP2 compared to CDK2-cyclin A are identified on the CDK2-cyclin A-p27 structure (PDB 1JSU). p27 is omitted from the SKP1-SKP2 panel for clarity. ${ }^{53}$

Table 2 SKP2 mutants display a range of binding affinities to cyclin A. $K_{d}$ values were determined by isothermal titration calorimetry.

\begin{tabular}{lll}
\hline Skp2 & Residue changes & $\begin{array}{c}\text { Cyclin A } \\
\mathrm{K}_{\mathrm{d}} \pm(\mathrm{nM})^{1}\end{array}$ \\
\hline Wild-type & None & $298 \pm 17$ \\
SKP2N_4A & L32A L33A S39A L41A & $5290 \pm 215$ \\
SKP2N_6A & W22A W24A L32A L33A S39A L41A & ND $^{2}$ \\
SKP2N_420 & N-terminal 20 residues deleted & $507 \pm 295$ \\
SKP2N_430 & N-terminal 30 residues deleted & ND \\
\hline
\end{tabular}

\footnotetext{
${ }^{1}$ Carried out at $25^{\circ} \mathrm{C}$ with a starting concentration of $10 \mu \mathrm{M}$ CDK2-cyclin A in the cell and $120 \mu \mathrm{M}$ SKP1-SKP2N in the syringe.

${ }^{2}$ Could not be determined. Experiments were carried out using a Malvern Microcal PEAQ-ITC. $K_{d}$ is the derived average of two independent biological replicates and SD of the population.
}

\section{The cyclin A SSMS site promotes cyclin A- SKP2 interaction in cellulo}

To evaluate the functional significance of the cyclin A SSMS site in cellulo, we first transfected full-length cyclin $A$, and full-length cyclin Amut5 into HEK293T cells (Figure 5(b)).
Immunoprecipitation exploiting the FLAG tag followed by western blotting revealed that whereas a robust interaction can be detected between authentic cyclin A and SKP2, introduction of the mut5 mutation abolished binding. Moreover, compared to wild type cyclin A, cyclin Amut5 has a reduced interaction with SKP1, CKS1 and 
(a)

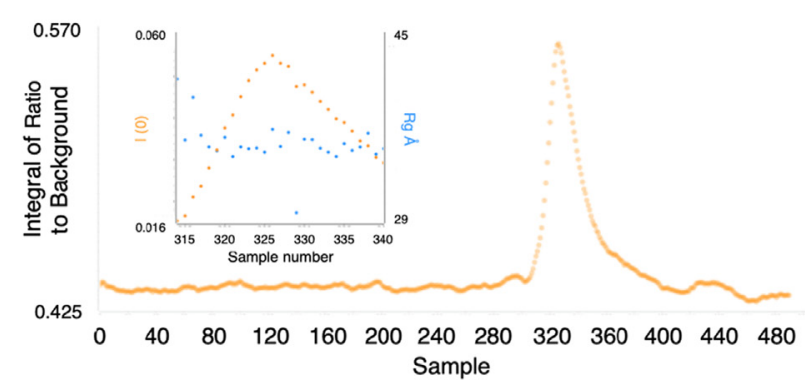

(c)

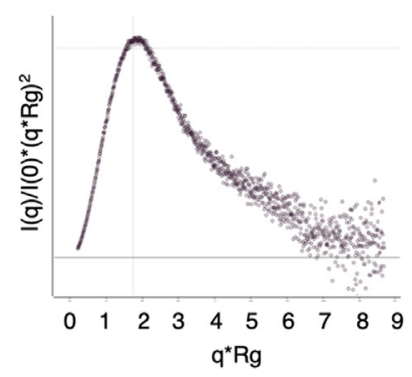

(d)

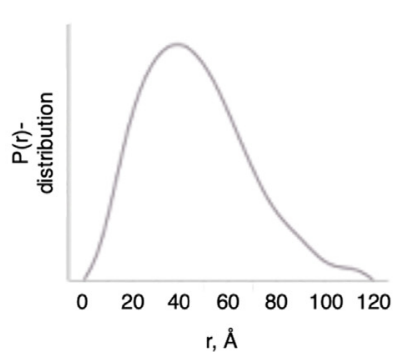

(b)

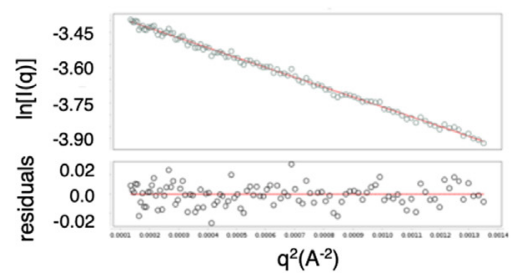

(e)

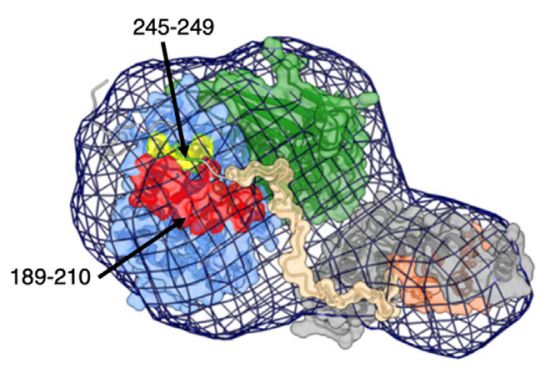

Figure 4. SAXS analysis of a CDK2-cyclin A-SKP1-SKP2 complex. Experimental plots for analysis of a SKP1 $(\triangle 38$ 43, $\triangle 69-83)-S K P 2(20-140-\Delta 60-74)$-CDK2-cyclin A complex by SAXS. (a) FPLC size-exclusion chromatogram and fraction analysis. (b) Guinier plot. The sample monodispersity and absence of aggregation was assessed by a linear fit of the Guinier plot $\left(\operatorname{In}(I)\right.$ vs $\left.q^{2}\right)$ (c) Kratky plot. The dimensionless Kratky plot $\left(q^{2} \times I(q) / I(0)\right)$ suggests a globular and relatively compact particle (maximum at $\mathrm{qRg}$ of 1.73 with a height of 1.1), with some indications of a multi-domain characteristic with only limited flexibility overall (indicated by the drop of the curve between qRg of 4-6). ${ }^{59}$ (d) Pairwise distance distribution. In agreement, the pairwise distance distribution $p(r)$ showed a bell shape indicating a fairly globular particle. A shoulder at $d=80-100 \AA$ indicated that a domain sticks out from the globular shape, whereas the tail at $d=105-120 \AA$ suggested the presence of a short flexible linker protruding from the particle. (e) SAXS-derived model for the CDK2-cyclin A-SKP1-SKP2N complex. CDK2 and cyclin A are colored as in Figure 1, the SKP2 F-box and SKP1 are colored salmon and grey respectively. Cyclin A residues 245-249 that includes the SSMS sequence is identified in lemon, and the SKP2 N-terminal sequence is modelled in khaki. Mesh represents the volume-adjusted (damfilt.pdb) converted into a map using molmap and visualized in ChimeraX. ${ }^{53}$

CDK2. These results demonstrate that the SSMS site makes a significant contribution to the stability of the pentameric CDK2-cyclin A-CKS1-SKP1SKP2 complex in a cellular setting. This site is not present in cyclin $E$ and helps to distinguish the abilities of these two cyclins to form stable complexes with SKP2.

To further assess the direct interaction between SKP2 and cyclin A and the non-direct interaction mediated by CKS1, N-terminally FLAG-tagged full-length authentic SKP2, SKP2_6A, SKP2_C2 and SKP2_6A_C2 were expressed in HEK293T cells to test their ability to bind to cyclin A (Figure 5(c)). The wild type SKP2 interacts robustly, but mutation of either the cyclin A-SKP2 (SKP2_6A mutant) or the CKS1-SKP2 interface (SKP2_C2 mutant) significantly decreases association. As expected, the cyclin A-SKP2 interaction is totally abolished when both binding sites are inactivated. Taken together these results support a model in which either the cyclin A-SKP2 or CKS1-SKP2 interaction can mediate complex formation, but that loss of both interaction sites abrogates binding.

\section{The cyclin A-SKP2 interaction recruits CDK2- cyclin A and affects p27 phosphorylation}

p27 can inhibit CDK1 and CDK2 bound to cyclins $A$ and $B$, or $A$ and $E$ respectively. Relief of $p 27$ inhibition and cell cycle progression results from CDK-dependent p27 phosphorylation at T187 and its subsequent targeting to the $\mathrm{SCF}^{\mathrm{SKP} 2} \mathrm{E} 3$ ubiquitin ligase. Our finding that p27 and the CAIM of SKP2 share overlapping footprints on cyclin A suggests that binding of p27 to a pentameric CDK1/2-cyclin A-CKS1-SKP1-SKP2 complex would displace the CAIM from its binding site on cyclin $A$, making it available to form alternative interactions. Because neither cyclin B nor cyclin E possesses a site that binds the CAIM, we would similarly expect the CAIM to be available in the pentameric CDK1/2-cyclin B-CKS1-SKP1-SKP2 and CDK1/2-cyclin E-CKS1-SKP1-SKP2 complexes. A potential role for the CAIM in these 
(a)
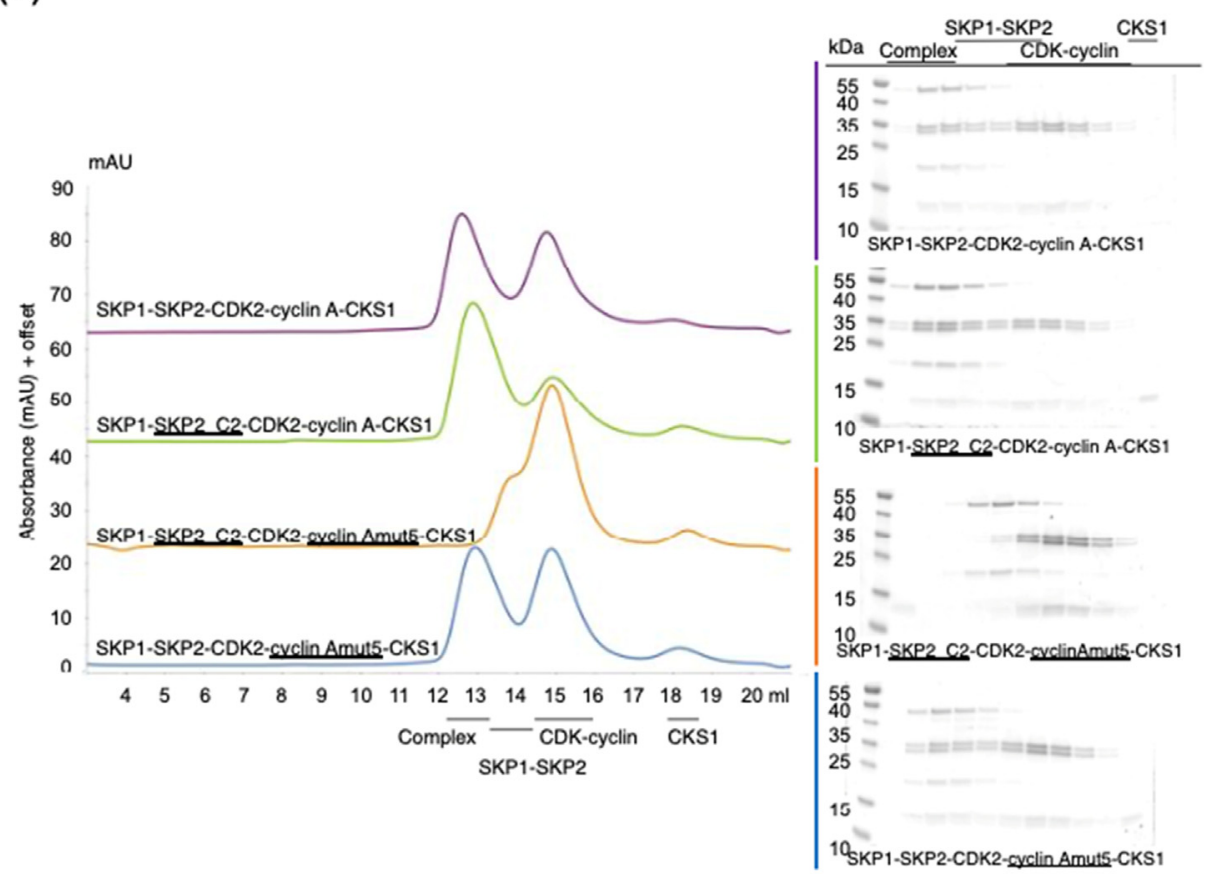

(b)

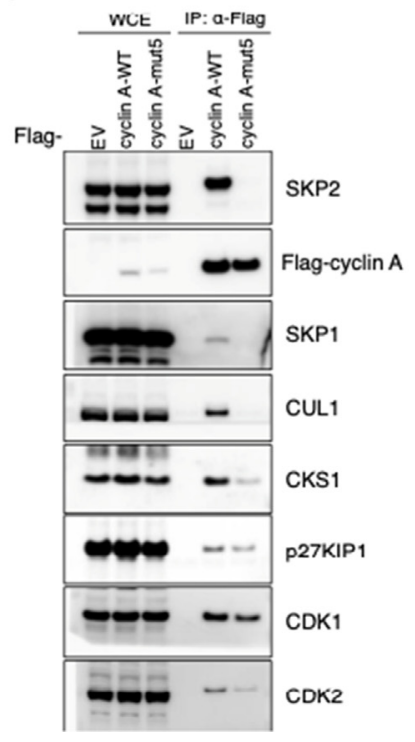

(c)

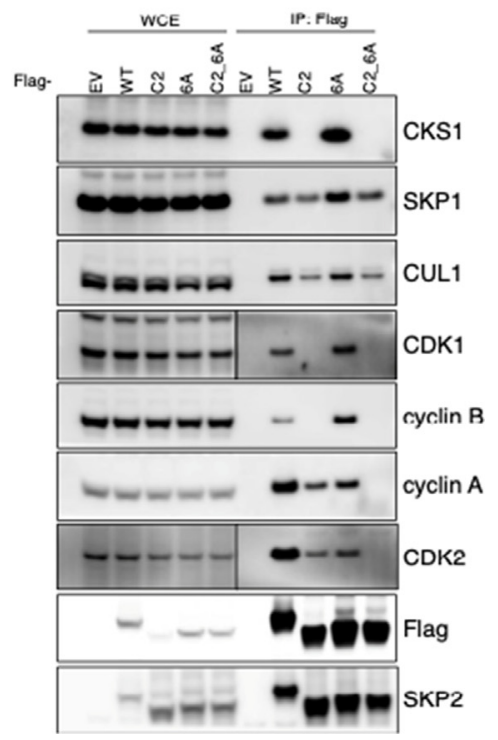

Figure 5. Characterization of the CDK2-cyclin A-SKP1-SKP2-CKS1 complex. (a) Size exclusion chromatography to assess the contribution of the SKP2-cyclin A and SKP2-CKS1 interfaces to generation of a stable pentameric complex. In the presence of an excess of CDK2-cyclin A-CKS1, SKP1-SKP2 forms a stable pentameric complex (magenta trace). Mutation of SKP2 at the CKS1 interface (SKP2_C2, green trace) or mutation of cyclin A at the SKP2 binding site (cyclin Amut5, blue trace) has little effect on complex stability. However, simultaneous mutation of both sites (orange trace) disassembles the complex into stable SKP1-SKP2 and CDK2-cyclin A-CKS1 sub-complexes. The accompanying SDS-PAGE analyses are provided in the color-matched boxes. Proteins were visualized using InstantBlue. Chromatograms are to the same scale but have been offset on the y-axis to aid comparison. Each chromatogram is representative of 2 replicates carried out using independently prepared proteins. (b) HEK293T cells were transfected with either an empty vector (EV) or FLAG-tagged cyclin A_WT or cyclin A_mut5. Twenty-four hours post-transfection, cells were collected for immunoprecipitation (IP) and immunoblotting. WCE, whole-cell extract. (c) HEK293T cells were transfected with either an empty vector (EV) or FLAG-tagged SKP2_6A, SKP2_C2, or SKP2_C2/6A. The experiment was performed as described in (b). 
complexes might be to recruit a further complex of CDK1/2-cyclin A (but not CDK1/2-cyclin B or CDK1/2-cyclin E). This additional copy might play a catalytic role in phosphorylating the $\mathrm{C}$ terminus of $\mathrm{p} 27$.

To test this model, we carried out SEC to evaluate whether a stable octameric complex might form when SBP-tagged CDK2-cyclinA is incubated with CDK2-cyclin A-CKS1-p27-SKP1-SKP2. Our results confirm the formation of such an octameric complex (Figure 6(a)), and further demonstrate that complex stability is dependent on the integrity of the SSMS site: no octameric complex is formed when SBP-tagged CDK2-cyclin Amut5 is incubated with CDK2-cyclin A-CKS1-p27-SKP1SKP2. We can also infer that the recruitment of the second CDK2-cyclin A module is dependent on p27 being present: SEC analysis of CDK2cyclin A-SKP1-SKP2-CKS1 in the presence of excess CDK2-cyclin A does not indicate formation of (CDK2-cyclin A) 2 -SKP1-SKP2-CKS1 complex (Figure 5(a), magenta trace).

To test whether the recruitment of a catalytic CDK2-cyclin A via the CAIM-cyclin A interaction impacts the kinetics of phosphorylation of p27, we next compared the rate of phosphorylation of p27 bound within complexes that incorporate catalytically inactive (CDK2 $\left.{ }^{\mathrm{D} 127 \mathrm{~N}}\right)$-cyclin $\mathrm{A}$ together with either CKS1-SKP1-SKP2 or CKS1-SKP1SKP2_6A. These complexes were incubated under initial rate conditions with either CDK2cyclin A or CDK2-cyclin E (Figure 6(b)). As expected, CDK2-cyclin $\mathrm{E}$ did not distinguish the two complexes, and p27 within each complex was phosphorylated at an equivalent initial rate. However, the initial rate of p27 phosphorylation was different when p27 was presented in the authentic complex than in the context of the SKP2-6A mutant. This result supports a model in which recruitment of CDK2-cyclin $A$ to the hexameric substrate results in a relatively longlived octameric complex. Such a complex would be primed for rapid initial phosphorylation. We hypothesize that the most plausible model to explain our results is that the slower turnover in steady state kinetics observed here may be explained by a relatively slow product release step.

\section{Discussion}

Cell cycle transition from $\mathrm{G} 1$ to $\mathrm{S}$ phase is tightly regulated by the sequential activation of CDK2 by cyclin $\mathrm{E}$ and then by cyclin A. p27 is both a CDK2 inhibitor and a CDK2 substrate and the balance of these activities ensures appropriate cell cycle progression. Signals to arrest the cell cycle during G1 are executed through p27-mediated CDK2 inhibition but, following checkpoint resolution, p27 is phosphorylated on T187 by CDK2-cyclin E and CDK2-cyclin A targeting it for ubiquitination by the SCF $^{\text {SKP2 }}$ E3 ubiquitin ligase and subsequent degradation by the proteasome. SKP2 levels are low in early G1 and increase late in G1. ${ }^{43}$

To characterize the molecular interactions that distinguish these various CDK2-containing complexes we set out to identify the SKP2 binding site on cyclin A. We show that the SKP2 binding site on cyclin A (the "SSMS site"), distinguishes cyclin A from cyclin $E$ and is distinct but overlapping with the cyclin A binding site for p27. We also note that the $S$. cerevisiae LP docking site is co-located on this surface of the cyclin NCBF. ${ }^{38}$ Indeed, a homology model of $\mathrm{Cln} 2$ indicates that Cln2 R109 (part of the R109, L112 and R113 LP docking site), is equivalent to M246 of the SSMS motif (Figure 6(c)). Our study also revealed that sequences that do not conform to the canonical RXL motif can bind to the cyclin recruitment site. This finding suggests that the recruitment site might be important for the cyclin localization of a larger number of proteins than might have been inferred by sequence analysis. Taken together, these results reveal that the $\mathrm{N}$-terminal cyclin box fold has an extended role in binding regulators. They also suggest that the cyclin A sequence and structure are finely tuned to optimize interactions with multiple binding partners. This site of direct interaction between SKP2 and cyclin A, in addition to the interaction between CKS1 and SKP2 shared with other CDK-cyclin modules, strengthens the cyclin A-SKP2 association and might explain why SKP2 was originally characterized as a component of a pentameric complex containing cyclin $\mathrm{A}$ and not cyclins B or E (Figure 6(d) "building"). ${ }^{1}$

Furthermore, we show that the cyclin A SKP2 binding site permits the recruitment of CDK-cyclin A but not CDK-cyclin B or cyclin E complexes to perform a catalytic function and promote the phosphorylation of p27 on T187, a modification that is a pre-requisite for its subsequent degradation by the SCF ${ }^{\text {SKP2 }}$ complex (Figure 6(d) "recruiting"). This activity is unique to cyclin A, whereas p27 recruitment to SKP2 is dependent on CKS1 and independent of the identity of the CDKcyclin to which it is bound. Thus, our results agree with a previous study in which it was shown that the ability of SKP2 to bind to cyclin A could be separated functionally from its ability to mediate p27 degradation. ${ }^{20}$ Such a model might also explain earlier studies in G1-enriched cell cultures, where it was found that CDK2-cyclin A overexpression was more efficient at promoting p27 ubiquitination than increasing the expression of CDK2-cyclin E and SKP2. ${ }^{44}$

However, it should also be noted that the SKP2 Nterminal sequence binds to other cell cycle regulators. Its occlusion within a pentameric SKP1-SKP2-CDK2-cyclin A-CKS1 complex may sequester it away from alternative interactions that might execute some of its functions or regulate its activity and thus perform an additional regulatory role. For example, cyclin A but not cyclin E competes with pRB and Cdh1 for binding to the 
SKP2 N-terminal sequence. ${ }^{18,19}$ This interaction between pRB and SKP2 has been proposed as a key mechanism mediating $\mathrm{G} 1$ arrest. ${ }^{21}$ Expression of cyclin $E$ in early $G 1$ will not antagonize this interaction, but as the cell moves through to late $\mathrm{G} 1$, cyclin A levels rise and SKP2 can now be assembled into an alternative complex with SKP1, CDK2, cyclin A and CKS1. This complex will both hinder the ability of pRB to stabilize p27 and prime SKP2 for its alternative role to mediate p27 degradation. We hypothesize that both events could relieve cell cycle control from $\mathrm{pRB}$ dependency and promote S-phase entry. The SKP2 N-terminal sequence is also phosphorylated (at S64, S72 and S75) and acetylated (at K68 and K71), and it might be hypothesized that anchoring the SKP2

(a)

(b)
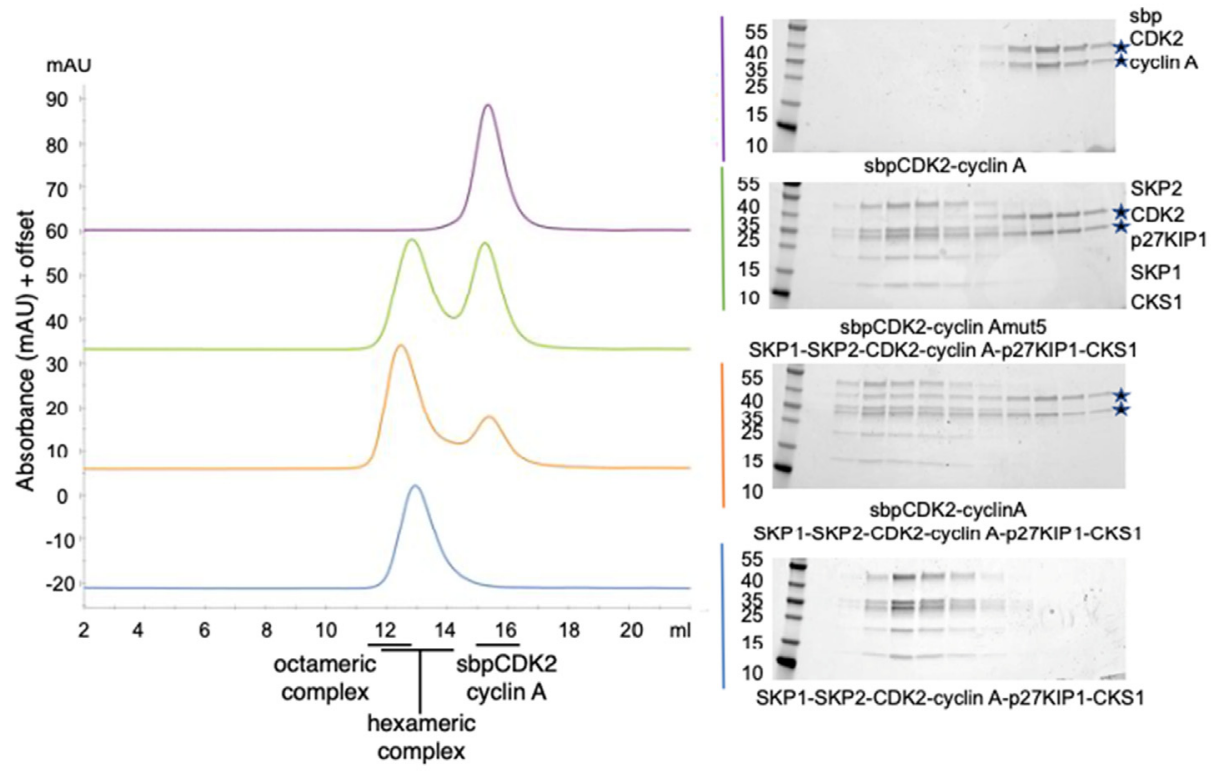

sbpCDK2-cyclinA
SKP1-SKP2-CDK2-cyclin A-p27KIP1-CKS1

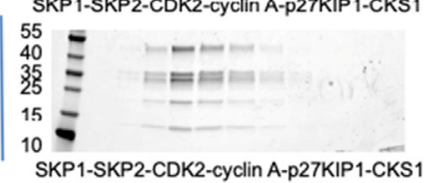

(c)

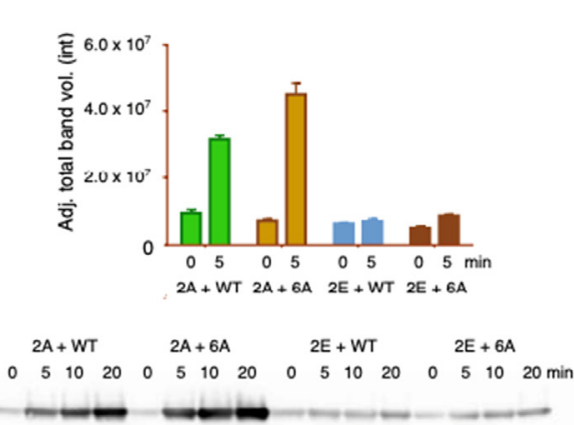

(c)

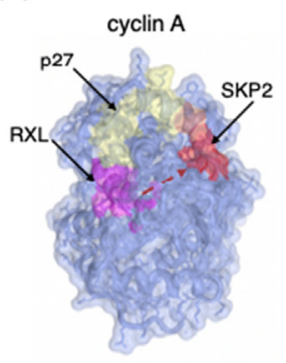

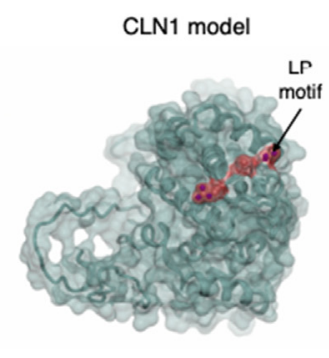

(d)

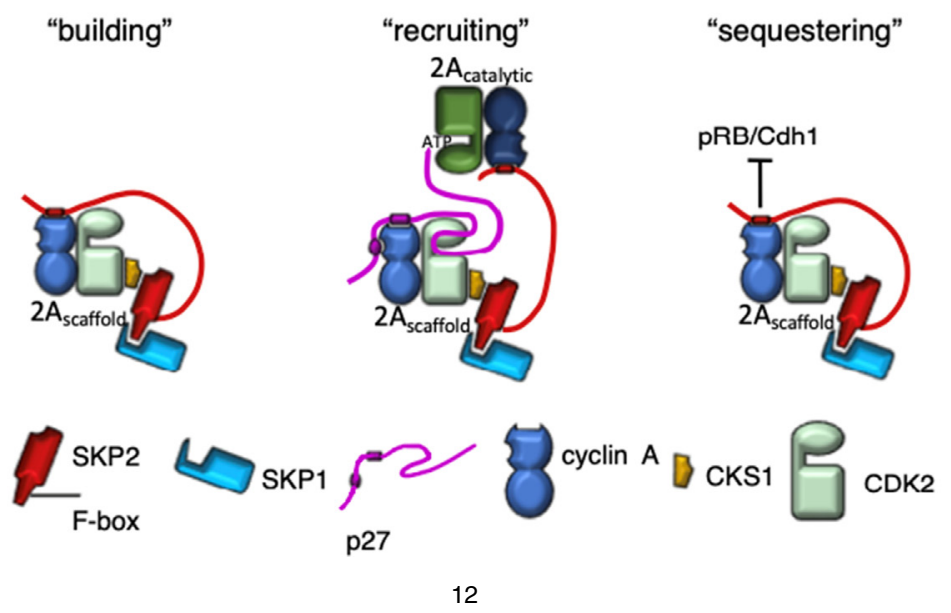


sequence on the surface of cyclin $A$ affects the accessibility of these residues to post translation modification, thereby modulating its activity (Figure 6(d), "sequestering"). Perturbations to these regulatory pathways may contribute in part to cancer progression seen in cells that overexpress cyclin $\mathrm{A},{ }^{1}$ as the interactions that mediate these regulatory pathways would be in direct competition with unphosphorylated p27 for binding to CDK2-cyclin A as has been previously proposed. ${ }^{20,45}$

The cyclin recruitment site that binds substrates and inhibitors containing an RXL motif is well characterized and illustrates the principle that a conserved site that is able to dock short peptide motifs with varying affinities offers a hub to integrate various signaling pathways. A similar principle orchestrates CDK9-cyclin $\mathrm{T}$ regulation by various activators and inhibitors that bind to overlapping surfaces on the C-terminal CBF. ${ }^{46,47}$ We demonstrate that the SKP2 and p27 cyclin A binding sites overlap permitting integrated regulation of activity. To what extent this principle will apply to any additional cyclin A protein binding sites and to what extent the SKP2 binding site or these other sites will further distinguish cyclins $A, B, D$ and $E$ awaits further identification and characterization of larger CDK-cyclin containing complexes.

\section{Materials and Methods}

\section{Protein expression and purification}

Full-length human GSTCDK2 co-expressed with S. cerevisiae GSTCAK1 from a pGEX-6P-1 vector $^{33,40}$ and untagged human cyclin A2 (residues 174-432), ${ }^{33}$ hexahistidine-tagged bovine cyclin A (residues 174-432) and full-length human CKS1 ${ }^{40}$ all cloned into the pET21d vector; and full-length p27 (residues 1-198, wild-type and T187A mutant), p27M (residues 1-106), p27S (residues 23-106), , and p27MS (residues 23-51, this paper) as GSTfusions from a modified pGEX-6P1 vector, were all expressed in E. coli BL21 (DE3) pLysS cells and purified as previously described. Briefly, GSTp27 constructs, bovine cyclin $\mathrm{A}_{\mathrm{His} 6}$ and human $\mathrm{CKS} 1_{\text {His6 }}$ were all purified by an initial affinity chromatography step exploiting their respective tags. The GST constructs were subsequently digested with $3 \mathrm{C}$ protease to cleave the tag. All constructs were subject to a second SEC step. N-terminal Avi-tagged CKS1 (residues 5-73) was expressed as an $\mathrm{N}$-terminally $\mathrm{His}_{6}$-tagged protein from the pET21d vector. It was purified by affinity chromatography, subsequent $3 \mathrm{C}$ protease cleavage to remove the $\mathrm{His}_{6}$ tag, then re-application on to the affinity column to remove any uncleaved protein

Figure 6. The cyclin A N-terminal cyclin box exploits an extended surface to interact with p27 and SKP2. (a) p27 association with CDK2-cyclin A can drive the formation of an octameric (CDK2-cyclin A) 2 -SKP1-SKP2-CKS1-p27 complex. p27 was first phosphorylated with an excess of CDK2-cyclin A and the ternary phosphorylated p27-CDK2cyclin A complex was then purified by SEC before being incubated with equimolar SKP1-SKP2 and an excess of CKS1. This hexameric complex purified by SEC (blue trace) was then incubated with either SBP-tagged CDK2cyclinA (orange trace) or SBP-CDK2-cyclinAmut5 (green trace). The SBP tag distinguishes these CDK2-cyclin A complexes by SEC from CDK2-cyclin A bound to p27. SBP-tagged CDK2-cyclin A forms an octameric complex but SBP-tagged CDK2-cyclin Amut 5 does not (compare the orange and blue traces, complex elution positions indicated below the chromatograms). sbpCDK2 and cyclin $A$ are identified by stars in the relevant gels. (b) The SKP2 CAIM recruits CDK-cyclin A to affect p27 phosphorylation. An intact cyclin A-SKP2 interaction recruits a catalytic CDK-cyclin module to phosphorylate p27 within a SKP1-SKP2-CDK2-cyclin A-CKS1-p27 complex. WT substrate, CDK2 ${ }^{\text {D127A }}$ -

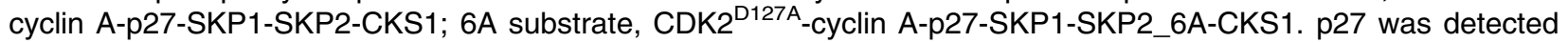
by western blot analysis using an anti phospho-p27 antibody, lower panel, representative time-course. Band intensity was revealed using ECL reagent and quantified using background adjusted total band volume with ImageLab software (BioRad). Graph is representative of assays carried out with three biological replicates of catalytically active CDK2-cyclin A and CDK2-cyclin E as control. (c) p27 and SKP2 binding to cyclin A. Cyclin A structure, highlighted in yellow, the p27 binding surface ((L255, Q290, R293, M294, L297 identified from PDB 1JSU), in red the SSMS site that binds to SKP2 (S244, S245, M246, S247) and in magenta the RXL recruitment site identified by residues I213 and W217. L297, mutation of which blocks both p27 and SKP2 binding, is colored orange. Dotted red arrow, the proposed SKP2 binding path. S. cerevisiae CLN2 shares the docking site that recognizes the RXL motif (the "HP region" ${ }^{38}$ with cyclin A but has an adjacent surface, defined by mutations R109, L112 and R113 that recognizes LP-motif containing substrates. These three sites form a continuous surface. Cyclin A M246 is equivalent to R109 in the CLN2 modelled structure. CLN1 model, highlighted in red, residues R109, L112, R113. The CLN2 structure was generated by implementing MODELLER ${ }^{60}$ in $\mathrm{HHpred}^{61}$ and then superimposed on the structure of cyclin A. (d) Proposed roles for the cyclin A-SKP2 interaction in regulating the cell cycle. The SKP2-cyclin A interaction promotes the incorporation of CDK-cyclin A complexes into SCF ${ }^{\text {KP2 }}$ ("building"). Incorporation of p27 into SKP1-SKP2-CDK1/2-cyclin A/B/E-CKS1 complexes generates complexes in which the CAIM can recruit a catalytic CDK1/2-cyclin A ("recruiting"). The cyclin A binding site on SKP2 overlaps with SKP2 binding sites for pRB and Cdh1 and so may alter the effective SKP2 concentration for alternative SKP2 regulators ("sequestering"). 
before a final SEC step. The global human cyclin A mutant sequence was synthesized by IDT, subcloned into an IDTsmart vector and then PCR amplified and inserted into pET21d to generate an untagged protein. Other human cyclin $\mathrm{A}$ mutants were prepared either starting from this template or the authentic sequence by site-directed mutagenesis (SDM). All CDK2-cyclin A complexes were purified following the method described in Brown et al. (1999). ${ }^{33}$ Briefly, GST-CDK2 was first loaded onto a glutathione affinity column and washed to baseline. This modified column was then used to affinity purify untagged cyclin $A$. The glutathione eluate (eluted with $20 \mathrm{mM}$ HEPES, NaCl $300 \mathrm{mM}$, DTT $1 \mathrm{mM}, 20 \mathrm{mM}$ glutathione, $\mathrm{pH} 7.6$ ) was incubated with $3 \mathrm{C}$ protease to release the CDK2-cyclin A from GST, which was subsequently further purified by SEC and glutathione affinity chromatography to remove any co-eluting GST. All steps from resuspension of the bacterial cell pellets were carried out in buffer containing $20 \mathrm{mM}$ HEPES pH 7.6, $300 \mathrm{mM} \mathrm{NaCl}, 1 \mathrm{mM}$ DTT. The CDK2 D127A mutant was introduced into a modified pET3d vector that co-expresses GST-CDK2, His-tagged bovine cyclin A and untagged p27 and the complex subsequently purified by sequential glutathione affinity and size exclusion chromatography steps. Human cyclin E1 (residues 96-378) was cloned into a modified pACEBac1 vector containing an $\mathrm{N}$ terminal MBP-3C tag. PCR of cyclin E1 (96-378) from a modified pGEX vector was conducted using primers: $5^{\prime}$-TTCCAGGGGCCCATGGGCATTATT GCACCATCCAGAGGCTCCC-3' (Forward) and 5'-GTTAGCAGCCACTAGTTCAGGCTTTCTTTG CTCGGGCTTTGTC-3' (reverse). pACEBac1_ MBP-3C-cyclin $E$ was then transformed into DH10_YFPEMBac E. coli for virus transposition, with appropriate clones selected through blue/ white screening. $20 \mathrm{ml}$ overnight cultures (in Insect Xpress media (Lonza)) of selected clones were grown. The virus was then extracted by isopropanol precipitation and amplified twice in Sf9 cells. For cyclin E expression, the virus was transfected into Sf9 cells at approximately 1.5 million cells $\mathrm{ml}^{-1}$ and grown for $72 \mathrm{~h}$ at $28^{\circ} \mathrm{C}$ with gentle mixing. Following incubation cells were harvested via centrifugation $\left(2600 \mathrm{~g}, 15 \mathrm{~min}, 4^{\circ} \mathrm{C}\right.$ ) and resuspended in $20 \mathrm{mM}$ HEPES pH 7.6, $300 \mathrm{mM} \mathrm{NaCl}$, $1 \mathrm{mM}$ DTT, supplemented with protease inhibitors. Cells were lysed by sonication and clarified by centrifugation $(100,000 \mathrm{~g})$. The soluble fraction was incubated with GST-CDK2 and then purified by affinity chromatography using Sepharose 4B GST resin beads (GE Healthcare) and subsequently eluted in $20 \mathrm{mM}$ HEPES, $300 \mathrm{mM} \mathrm{NaCl}$, $1 \mathrm{mM}$ DTT, $20 \mathrm{mM}$ glutathione, $\mathrm{pH}$ 7.6. Following $3 \mathrm{C}$ protease treatment CDK2-cyclin $\mathrm{E}$ was buffer exchanged using SEC (Superdex 200 10/300) and then loaded onto a GST-trap column $(5 \mathrm{ml}$, GE Healthcare) and then onto an amylose resin
(New England Biolabs). A final SEC step (Superdex $20010 / 300$ ) was performed in order to remove any GST and MBP contamination. Fulllength SKP2 was co-expressed as a GST fusion (cleavable with $3 \mathrm{C}$ protease) with untagged fulllength SKP1 from a modified pET3d backbone. Each protein was expressed from separate transcripts. A GSTSKP2N-SKP1 complex was expressed from a pGEX-6P-1 vector in E. coli BL21 (DE3) pLysS cells (Agilent Technologies). This construct encodes SKP2 residues 1-140 (SKP2N) and SKP1 (residues 1-163, with internal deletion of residues $38-43$ and $69-83$ as described by Schulman et al. ${ }^{28}$ The two proteins are translated from a single transcript, the SKP1 initiating at an internal ribosome binding site. This construct was used as the template to make the SKP1-SKP2N_4A (L32A, L33A, S39A and L41A), and SKP1-SKP2N_6A (SKP2_4A and W22A and W24A) mutants by SDM and the constructs starting at F20 (SKP1-SKP2N_ $\Delta 20)$ and at S30 (SKP1-SKP2N_ $\triangle 30$ ) by PCR. $\bar{N}$-terminal SKP2 peptides were either synthesized (FTWGWDSSKTSELLSGMGVSALE) and modified by $\mathrm{N}$-terminal acetylation and $\mathrm{C}$-terminal amidation (Severn Biotech Ltd) or generated by SDM (SKP2 residues 20-51 and 20-64) starting with the GSTSKP2N-SKP1 expression construct. For the HTRF assay presented in Supplementary Figure S6, the SKP2 C-terminal sequence was mutated by SDM to replace F393 with glycine and serine (SKP2 F393GS) and co-expressed and purified with SKP1. The construct using the pGEX-6P-1 backbone is based on that described in Schulman et al., that encodes SKP2 residues 89-424 and SKP1 (residues 1-163, with internal deletion of residues 38-43 and 69-83). ${ }^{28}$ The SKP2 phosphorylation site mutant (S64A) was prepared by SDM using the GSTSKP2-SKP1 co-expression construct in the modified pET3d backbone described above that encodes full-length SKP2. E2 R1 and all the SKP1-SKP2 expression constructs described above were purified by sequential glutathione affinity and SEC chromatography steps in $20 \mathrm{mM}$ HEPES, $300 \mathrm{mM} \mathrm{NaCl}, 1 \mathrm{mM}$ DTT. The p107 substrate peptide was prepared as described in 40. Briefly, the protein was expressed as a GST-fusion from the pGEX6P-1 backbone in E. coli Rosetta (DE3) pLysS cells at $20^{\circ} \mathrm{C}$ overnight subsequent to induction with $0.1 \mathrm{mM}$ IPTG. It was first purified by a glutathioneaffinity step and then subsequent to tag removal following incubation with $3 \mathrm{C}$ protease further purified on a MonoS ion-exchange (GE Healthcare) column equilibrated into $25 \mathrm{mM}$ MES $\mathrm{pH}$ 6.5. The peptide was stepped into $25 \mathrm{mM}$ MES, $0.5 \mathrm{M} \mathrm{NaCl}, \mathrm{pH} 6.5$ for rapid peptide release. Fractions containing the peptide were confirmed by SDS-PAGE, pooled, concentrated to a final peptide concentration between 1 and $2 \mathrm{mg} \mathrm{ml}^{-1}$ and then flash frozen and stored at $-80{ }^{\circ} \mathrm{C}$ in $100 \mu \mathrm{l}$ aliquots. Protein concentrations 
were measured by $A_{280} \mathrm{~nm}$ on a Nanodrop2000 (Thermo Fisher Scientific) using calculated extinction coefficients (http://web.expasy.org/protparam/).

\section{Isothermal titration calorimetry (ITC)}

ITC was performed using a Malvern Microcal PEAQ-ITC. Proteins were equilibrated in HEPES $20 \mathrm{mM} \mathrm{pH}$ 7.97, NaCl $300 \mathrm{mM}$, TCEP $0.5 \mathrm{mM}$. The concentrations of CDK2-cyclin A or cyclin A in the cell and SKP1-SKP2N in the syringe for each experiment are provided in the accompanying Table Legends. Protein concentrations (for this and other biophysical techniques) were measured by $\mathrm{A}_{280} \mathrm{~nm}$ on a Nanodrop2000 (Thermoscientific) using calculated extinction coefficients (http:// web.expasy.org/protparam/). Experiments (including ligand dilution controls) were carried out at $25{ }^{\circ} \mathrm{C}$. The titrations consisted of 20 injections, an initial injection of $0.5 \mu \mathrm{l}$ and then 19 injections of $19.5 \mu \mathrm{l}$ with $180 \mathrm{~s}$ between injections. The stirring speed in the reaction cell was $1000 \mathrm{rpm}$. Data were analyzed using non-linear least squares regression using MicroCal PEAQ analysis software. Drifts in the baseline were corrected during data analysis. As detailed in Table 1, for characterization of authentic interactions and mutants that were characterized further by other orthogonal techniques, $K_{d}$ values are presented as the average of two independent biological replicates and SD of the population. For other mutants, $K_{d}$ values were derived from one measurement where the error represents the fitting error directly derived from the thermogram. Significance was calculated at $p<0.05$ using a Student's t-test.

\section{Analytical size-exclusion chromatography}

Size exclusion chromatography was performed using a Superdex 200 (10/300, GE Healthcare) on an AKTA Pure at controlled temperature of $4{ }^{\circ} \mathrm{C}$, pre-equilibrated in HEPES $20 \mathrm{mM} \mathrm{pH} \mathrm{7.5,} \mathrm{NaCl}$ $300 \mathrm{mM}$, DTT $1 \mathrm{mM}$. Each complex was reconstituted in vitro in $0.4 \mathrm{ml}$ of HEPES $20 \mathrm{mM}$ $\mathrm{pH} 7.5, \mathrm{NaCl} 300 \mathrm{mM}$, DTT $1 \mathrm{mM}$ and glycerol $10 \%$, and incubated for $1 \mathrm{~h}$ at $4{ }^{\circ} \mathrm{C}$ prior to elution. The complexes were combined at a molar ratio of 1:1.5 corresponding to SKP1-SKP2 $(4 \mu \mathrm{M})$ :CDK2cyclin $\mathrm{A} / \mathrm{E} /$ mut5 $(6 \mu \mathrm{M})$ in Figure $1(\mathrm{a})$; a molar ratio of 1:1.5:2.25 corresponding to SKP1-SKP2/ C2 $(4 \quad \mu \mathrm{M})$ :CDK2-cyclin $\mathrm{A} /$ mut5 $(6 \quad \mu \mathrm{M})$ :CKS1 $(9 \mu \mathrm{M})$ in Figure $5(\mathrm{a})$, and SKP1-SKP2/C2 $(4 \mu \mathrm{M})$ : CDK-cyclin $(6 \mu \mathrm{M})$ :CKS1 $(9 \mu \mathrm{M})$ in S6B; a molar ratio $1: 1.5$ of the pre-purified hexameric complex (SKP1-SKP2-CDK2-cyclinA-p27-CKS1) (4 $\mu \mathrm{M})$ and SBP-tagged CDK2-cyclin A/mut5 $(6 \mu \mathrm{M})$ in Figure $6(\mathrm{a})$ and a molar ratio of 1:1.5 corresponding to SKP1-SKP2 $(4 \mu \mathrm{M})$ :CDK2 or cyclin $A(6 \mu \mathrm{M})$ in Figure $\mathrm{S} 1(\mathrm{~A})$. For reconstitution of the p27-containing complex the ratio was adjusted to 1:1.5:2.25:3.375 using SKP1-SKP2
$(4 \mu \mathrm{M})$ :CDK2-cyclin A $(6 \mu \mathrm{M})$ :p27S $(9 \mu \mathrm{M})$ :CKS1 $(13.5 \mu \mathrm{M})$ in Figure $S 6(C)$. Calibration of the Superdex 200 (10/300) was performed using $\beta$ Amylase $(200 \mathrm{kDa})$, albumin $(66 \mathrm{kDa})$ and carbonic anhydrase (29 kDa) protein molecular weights standards (Sigma Aldrich). Corresponding fractions for each chromatogram between 12 and $18 \mathrm{ml}$ with an interval of $0.5 \mathrm{ml}$ were analyzed by SDS-PAGE (Biorad) and InstantBlue coomassie staining (Expedeon).

\section{Surface plasmon resonance (SPR)}

SPR analysis was carried out using a Biacore S200 (GE Healthcare). The sensor chip CM5 surface was functionalized using an anti GST tag antibody by amine-coupling. Routinely, ligand capture on flow cell number 2 was carried out for $60 \mathrm{~s}$ followed by multiple buffer injection to remove the excess of inactive protein, and the intensity was normalized against flow cell number 1 (GST control as ligand to correct for non-specific interactions and bulk effects). Experiments were performed in HEPES $20 \mathrm{mM} \mathrm{pH7.4,} \mathrm{NaCl} 150 \mathrm{mM}$ Tween20 $0.01 \%$ at $20{ }^{\circ} \mathrm{C}$. GSTp27M was captured on the functionalized surface of flow cell 2 for $60 \mathrm{~s}$ at a flowrate of $5 \mu \mathrm{min}^{-1}$. CDK2-cyclin A wild-type and mutants were then titrated as analytes using a 4-fold dilution series and then the chip was regenerated using glycine $\mathrm{pH} 2.1$ for $60 \mathrm{~s}$. The sample contact time (60 s) was measured using high performance settings followed by $240 \mathrm{~s}$ of dissociation time at $30 \mu \mathrm{l} \mathrm{min}^{-1}$. Two independent (biological replicate) experiments were performed. Sensorgrams were analyzed using Biacore S200 Evaluation Software 1.0 (GE Healthcare) and the curves were fitted using 1:1 binding kinetics.

\section{Homogenous time-resolved fluorescence assay (HTRF)}

In this format the HTRF assay measures the interaction between GSTSKP2-SKP1 and Nterminal Avi-tagged CKS1. Optimized buffer and detection reagent conditions were $3 \mathrm{nM}$ Tb-antiGST antibody, $50 \mathrm{nM}$ SA-XL665, in $50 \mathrm{mM}$ HEPES $\mathrm{pH} 7.5,100 \mathrm{mM} \mathrm{NaCl}, 1 \mathrm{mM}$ DTT, $0.1 \mathrm{mg} \mathrm{ml}^{-1}$ BSA, 1\% DMSO. Experiments were carried out at GSTSKP2-SKP1 or GSTSKP2_C2SKP1 concentrations of 1,5 or $25 \mathrm{nM}$ into which $\mathrm{N}$-AviCKS1 was titrated from 1 to $1000 \mathrm{nM}$. Reactions were set up in a volume of $20 \mu \mathrm{l}$ in 384 well plates and incubated for $30 \mathrm{~min}$ at $4{ }^{\circ} \mathrm{C}$. Samples were excited using a wavelength of $337 \mathrm{~nm}$ and emission spectra measured at $620 \mathrm{~nm}$ and $665 \mathrm{~nm}$ using a PHERAstar plate reader (BMG Labtech). Binding curves were plotted using GraphPad Prism 6 from which the $\mathrm{K}_{\mathrm{d}} \mathrm{s}$ were determined. 


\section{Differential scanning fluorimetry}

A protein/Sypro orange mix containing $5 \mu \mathrm{M}$ protein and a 1:5000 dilution of dye in DMSO (as supplied by Sigma Aldrich) was prepared just before plate setup in $20 \mathrm{mM}$ HEPES $\mathrm{pH}$ 7.5, $150 \mathrm{mM} \mathrm{NaCl}$ and $0.5 \mathrm{mM}$ TCEP. $14.5 \mu \mathrm{L}$ of the protein/Sypro orange mix was aliquoted into 384well plates and sealed. Each experiment was done in triplicate. Thermal melting experiments were carried out using a QuantStudio Real Time PCR machine (Applied Biosystems). The plates were first equilibrated at $25^{\circ} \mathrm{C}$ for $2 \mathrm{~min}$ in the PCR machine before starting the thermal melting experiment upon which the plates were heated at $0.05{ }^{\circ} \mathrm{C} \mathrm{sec}{ }^{-1}$ from 25 to $99^{\circ} \mathrm{C}$. The fluorescence intensity was recorded using the ROX filters under continuous collection. Raw fluorescence data were extracted from the QuantStudio Real-Time PCR software and analysed using the Applied Biosystems Protein Thermal Shift software. Calculated derivative $T_{m}$ values for each of the three repeats were extracted and the average values calculated.

\section{Hydrogen-deuterium exchange mass spectrometry}

HDX-MS experiments were carried out using an automated HDX robot (LEAP Technologies, Fort Lauderdale, FL, USA) coupled to an M-Class Acquity LC and HDX manager (Waters Ltd., Wilmslow, Manchester, UK). Analysis of the CDK2-cyclin A-p27 and CDK2-cyclin A-SKP1$\triangle 20$ SKP2 complexes was carried out separately and so were plotted individually. All samples were diluted to $10 \mu \mathrm{M}$ in equilibration buffer $(50 \mathrm{mM}$ potassium phosphate buffer $\mathrm{pH}$ 7.4) prior to analysis. $30 \mu \mathrm{l}$ sample was added to $135 \mu \mathrm{l}$ deuterated buffer $(10 \mathrm{mM}$ potassium phosphate buffer $\mathrm{pH} 7.4$ ) and incubated at $4{ }^{\circ} \mathrm{C}$ for $0.5,1,2$, 10 or $60 \mathrm{~min}$. Following the labelling reaction, samples were quenched by adding $50 \mu \mathrm{l}$ of the labelled solution to $100 \mu$ l quench buffer $(50 \mathrm{mM}$ potassium phosphate, $0.05 \%$ DDM pH 2.2) giving a final quench $\mathrm{pH} \sim 2.5 .50 \mu \mathrm{l}$ of quenched sample (ca. 24 pmol) were passed through an immobilized Ethylene Bridged Hybrid (BEH) pepsin column (Waters Ltd., Wilmslow, Manchester, UK) at $500 \mu \mathrm{min}^{-1}\left(20{ }^{\circ} \mathrm{C}\right)$ and a VanGuard Pre-column Acquity UPLC BEH C18 $(1.7 \mu \mathrm{m}, 2.1 \mathrm{~mm} \times 5 \mathrm{~mm}$, Waters Ltd., Wilmslow, Manchester, UK) for $3 \mathrm{~min}$ in $0.3 \%$ formic acid in water. The resulting peptic peptides were transferred to a C18 column $(75 \mu \mathrm{m} \times 150 \mathrm{~mm}$, Waters Ltd., Wilmslow, Manchester, UK) and separated by gradient elution of $0-40 \% \mathrm{MeCN}$ $\left(0.1 \% \mathrm{v} / \mathrm{v}\right.$ formic acid) in $\mathrm{H}_{2} \mathrm{O}(0.3 \% \mathrm{v} / \mathrm{v}$ formic acid) over $7 \mathrm{~min}$ at $40 \mu \mathrm{l} \mathrm{min}{ }^{-1}$. Trapping and gradient elution of peptides was performed at 0 。 C. The HDX system was interfaced to a Synapt G2Si mass spectrometer (Waters Ltd., Wilmslow,
Manchester, UK). HDMSE and dynamic range extension modes (Data Independent Analysis (DIA) coupled with IMS separation) were used to separate peptides prior to CID fragmentation in the transfer cell. ${ }^{49} \mathrm{HDX}$ data were analyzed using PLGS (v3.0.2) and DynamX (v3.0.0) software supplied with the mass spectrometer. Restrictions for identified peptides in DynamX were as follows: minimum intensity: 1000, minimum products per MS/ MS spectrum: 5, minimum products per amino acid: 0.3 , maximum sequence length: 25 , maximum ppm error: 5 , file threshold: $3 / 3$. Following manual curation of the data, PAVED was used ${ }^{42}$ to determine areas of the protein showing significant differences in exchange between the states. Briefly, for each time point and each amino acid residue in the sequence, the mean relative fractional uptake was calculated from all peptides covering that amino acid where the amino acid is not at the $\mathrm{N}$ terminus of the peptide. Analysis of variance and post-hoc Tukey tests were used to determine significant differences $(p<0.05)$. For each region of significant difference, a representative peptide was chosen for the following discussion.

\section{Small angle X-ray scattering and model building}

The SKP1 $(\Delta 38-43, \Delta 69-83)-S K P 2$ (20-140- $\Delta 60$ 74)-CDK2-cyclin A complex was purified by size exclusion chromatography in $20 \mathrm{mM}$ HEPES $150 \mathrm{mM} \mathrm{NaCl} 1 \mathrm{mM}$ DTT $\mathrm{pH} 7.0$ at a final concentration of $5 \mathrm{mg} \mathrm{m}^{-1}$. On the BL21 beamline at the Diamond Light Source the complex was injected though a HPLC-size exclusion column collecting 490 individual SAXS frames (Supplementary Table 3). Fractions were analyzed at $10{ }^{\circ} \mathrm{C}$, using an X-ray wavelength of $12.4 \mathrm{KeV}$ at a detector distance of $4.014 \mathrm{~m}$, resulting in the momentum transfer range of $0.004 \AA^{-1}<q<0.4420 \AA^{-1}$. The average and the median were calculated from peak fractions subtracted from selected buffer frames of the column void. Data were scaled, merged and analyzed using Scatter $3{ }^{50}$ To generate the model, a SKP2 fragment (28-45) was built in MOE (2018.1), energy minimized and subject to conformer generation. A randomly selected conformer was docked to cyclin A (PDB 1VIN) in MOE using the MRAIL and SSMS motifs as targeted binding sites. Best solutions were subject to molecular dynamics for refinement and the resulting model with the best interface area (as analyzed by PDBePISA (v1.52)) was used as a starting model to extend the peptide $\mathrm{N}$-terminally to the full fragment 17-45 (ATSFTWGWDSSKTSELLSGMGVSALE $\mathrm{KEE})$. The extended model was refined in molecular dynamics simulation. A 35 dummy atom linker was introduced to connect the peptide to SKP1-SKP2(Fbox) (PDB 2AST). Ab initio molecular bead models were generated using DAMMIF. ${ }^{51}$ Rigid body modelling carried out within CORAL ${ }^{52}$ 
gave a Chi square fitting of 1.45 with the experimental data. The volume-adjusted ('filtered') average of these models (damfilt.pdb) was converted into a map using molmap and visualized in ChimeraX. ${ }^{53}$

\section{Crystallization and structure determination}

CDK2-cyclin Amut5 was concentrated to $5.6 \mathrm{mg} \mathrm{ml}^{-1}$ in HEPES $20 \mathrm{mM} \mathrm{pH} \mathrm{7.6,} \mathrm{NaCl}$ $150 \mathrm{mM}$, DTT $1 \mathrm{mM}$. Crystallization trials were set up at $4{ }^{\circ} \mathrm{C}$ using the sitting drop vapor diffusion method. Crystals were grown in condition B10 of the JCSG + crystallization screen (Molecular Dimensions) composed of $200 \mathrm{mM} \mathrm{K} / \mathrm{Na}$ Tartrate, 20\% PEG 3350. They were subsequently harvested using fibre loops and flash-cooled in liquid nitrogen, using 20\% PEG400 as cryoprotectant. X-ray diffraction data were collected at $100 \mathrm{~K}$ at the Diamond Light Source (Didcot, UK), beamline 104-1 operating at a wavelength of $0.92 \AA$. The automatic molecular replacement program MOLREP (within the CCP4 suite. ${ }^{54}$ was used to calculate molecular replacement solutions and the correlation coefficients. Model building was done using Coot. ${ }^{55}$ The final model has $0.3 \%$, $1.8 \%$ and $1.4 \%$ defined as Ramachandran, sidechain or RSRZ outliers (wwPDB X-ray structure validation report). Structural figures were prepared using the CCP4 molecular graphics program CCP4MG. ${ }^{56}$ Statistics generated from X-ray crystallography data processing, refinement, and structure validation are displayed in Supplementary Table S1.

\section{Kinase assays}

Non-phosphorylated p27 substrate complexes were assembled by incubating co-expressed CDK2 ${ }^{\mathrm{D} 127 \mathrm{~A}}$-cyclin A-p27 with an excess of either SKP1-SKP2-CKS1 or SKP1-SKP2_6A-CKS1 and subsequently purified using a Superdex 200 column equilibrated in $20 \mathrm{mM}$ Tris- $\mathrm{HCl} \mathrm{pH} \mathrm{7.8,}$ $300 \mathrm{mM} \mathrm{NaCl}, 0.5 \mathrm{mM}$ TCEP, 5\% glycerol. Kinase assays were carried out in SEC buffer supplemented with $0.75 \mathrm{mM}$ ATP, $2 \mathrm{mM} \mathrm{MgCl}_{2}$, $0.1 \mathrm{mg} \mathrm{ml}^{-1}$ BSA. $2.5 \mathrm{nM}$ CDK2-cyclin A was incubated with a $2170 \mathrm{nM}$ of substrate complex at $15{ }^{\circ} \mathrm{C}$ and the reaction was stopped after 5,10 and $20 \mathrm{~min}$ by addition of Laemmli buffer at $95^{\circ} \mathrm{C}$ for $2 \mathrm{~min}$, and subsequently analyzed by SDSPAGE (Criterion, Biorad). CDK2-cyclin E was initially assayed using the same conditions and then subsequently assayed at 0 and $5 \mathrm{~min}$ with its concentration adjusted to $5 \mathrm{nM}$. Protein samples were transferred and western blotted using anti phospho-T187 p27 antibody (Merck, 06-996). Band intensity was revealed using ECL reagent (BioRad) and quantified using background adjusted total band volume with ImageLab software (BioRad). Assays were carried out with three biological replicates of catalytically active CDK2-cyclin $\mathrm{A}$ and CDK2-cyclin $\mathrm{E}$ as control.
Other kinase assays were carried out as described. ${ }^{40}$ Briefly, phosphorylated CDK2-cyclin $A$, selected CDK2-cyclin A mutants, CDK2-cyclin A-SKP1-SKP2 (full-length SKP1 and full-length wild-type SKP2 and S64A mutant) and CDK2cyclin A-p27M (residues 1-106) were assayed using the ADP-Glo ${ }^{T M}$ assay (Promega) using a p107 peptide (peptide SPIK + RXL: IGSGVLFQG PLGSMHPRVKEVRTDSGSLRRDMQPLSPISVH ER WSSATAGSAKRRLFGEDPPKEMLMDKW) ${ }^{40}$ essentially as described by the manufacturers. In brief, in a final volume of $5 \mu$, reactions were carried out at room temperature in $40 \mathrm{mM}$ Tris- $\mathrm{HCl} \mathrm{pH} \mathrm{7.5,}$ containing $20 \mathrm{mM} \mathrm{MgCl}_{2}, 0.1 \mathrm{mg} \mathrm{ml}^{-1} \mathrm{BSA}$, and 1.5 nM CDK2-cyclin A or CDK2-cyclin A-SKP1SKP2 complex. Peptide dilutions $(94-0 \mu \mathrm{M})$ were added to the CDK-cyclin solution and the reactions were initiated by the addition of 75 or $25 \mu \mathrm{M}$ ATP (as indicated in Supplementary Table S2). All activity assays were performed in duplicate or triplicate (as indicated in Supplementary Table S2) in white low volume 384-well plates using a PheraStar plate reader (BMG). $\mathrm{K}_{\mathrm{m}}$ and $\mathrm{V}_{\max }$ values were obtained by fitting the data to the equation $\mathrm{v}=\mathrm{V}_{\text {max }} /$ $\left(1+\left(K_{m} /[S]\right)\right.$ using PRISM (GraphPad).

\section{Cell-based studies}

HEK293T (female) cells were propagated in DMEM supplemented with $10 \%$ fetal bovine serum (FBS) (Corning Life Sciences) and 1\% penicillin/streptomycin/L-glutamine (Corning Life Sciences). Cyclin A, cyclin A_mut5, SKP2_6A, SKP2_C2, and SKP2_6A_C2 cDNAs were inserted into a modified pcDNA3.1 vector containing an N-terminal FLAG tag by subcloning. SKP2 cDNA was inserted into the pcDNA3.1 vector containing an N-terminal FLAG/Strep tag. HEK293T cells were transiently transfected using polyethylenimine. Twenty-four hours after transfection, HEK293T cells were collected and lysed with lysis buffer (50 mM Tris $\mathrm{pH}$ 8.0, $150 \mathrm{mM} \mathrm{NaCl}, 10 \%$ glycerol, $1 \mathrm{mM}$ EDTA, $50 \mathrm{mM}$ $\mathrm{NaF}$, and $0.2 \%$ NP-40) supplemented with protease and phosphatase inhibitors. Lysates were then immunoprecipitated with anti-FLAG antibody conjugated to agarose. Elution of the immunoprecipitation from the anti-FLAG agarose resin was carried out with 3xFLAG peptide. Immunoblotting was performed as previously described. ${ }^{57}$ Briefly, samples were resolved under denaturing and reducing conditions using 4-12\% Bis-Tris gels (NuPAGE ${ }^{\circledR}$ ) and transferred to a PDVF membrane (Immobilon-P, Millipore). Membranes were blocked with $5 \%$ nonfat dried milk, then incubated with primary antibodies overnight at $4^{\circ} \mathrm{C}$. After washing the membranes, secondary antibodies coupled with horseradish peroxidase were applied (Amersham-GE). Immunoreactive bands were visualized by enhanced chemiluminescence reagent (Thermo Fisher Scientific) and signal was acquired using ImageQuant LAS 400 (GE). The fol- 
lowing antibodies were used for immunoblotting: anti-CUL1 (Santa Cruz Biotechnology, sc-11384, 1:500), anti-FLAG (Sigma, F7425, 1:2000), SKP2 (Invitrogen, 32-3300, 1:1000). Antibodies against CKS1, SKP1, cyclin A, cyclin B, CDK1 and CDK2 are homemade from the Michele Pagano lab.

\section{Accession Numbers}

The coordinates and the structure factors have been deposited in the Protein Data Bank under ID code 6SG4. Other data are available from the corresponding author upon reasonable request.

\section{Acknowledgements}

X-ray crystallography and SAXS was carried out with the support of Diamond Light Source on beamlines 104-1 (proposal MX13587) and BL21 (sm16970-2) respectively. We thank N. Schueller, M. Hoellerer and H. Ruddick (University of Oxford) for preparation and initial construct characterization and for assistance with preparation of the mutants; K. Cole, M. Martin, C. Jennings and $A$. Wittner for assistance with the SEC, ITC, mass spectrometry and cell-based studies respectively. $\mathrm{H}$. Waller and $\mathrm{O}$. Davies provided invaluable assistance to carry out the ITC and SEC-MALLS. B. Hao (U. Connecticut) for providing the SKP1-SKP2 co-expression plasmid. This research was supported by the Wellcome Trust (Grant Reference 063551); MRC (Grant References G0901526 and MR/N009738/1), Cancer Research UK (Grant Reference C2115/ A21421), Newcastle Cancer Centre and the JGW Patterson Foundation and the BBSRC (Grant Reference BB/M012573/1). The LEAP sample handling robot used in the HDX-MS work was a kind donation from Waters UK. M.P. is an Investigator with the Howard Hughes Medical Institute and funded by grants from the National Institutes of Health to M.P. (GM136250 and CA76584). Research by S.A. reported in this publication was supported by the King Abdullah University of Science and Technology (KAUST).

\section{Additional information}

M.P. has financial interests in CullGen Inc. and Kymera Therapeutics. M.P. is on the SAB of CullGen Inc. and Kymera Therapeutics. M.P. is a consultant for CullGen Inc., Kymera Therapeutics, Exo Therapeutics, and SEED Therapeutics. J.R. is a current employee of Astex Pharmaceuticals. The other authors declare no competing interests. The authors declare no competing financial interest. Some work in the authors' laboratory is supported by a research grant from Astex Pharmaceuticals.

\section{Appendix A. Supplementary material}

Supplementary data to this article can be found online at https://doi.org/10.1016/j.jmb.2020. 166795.

Received 26 October 2020; Accepted 28 December 2020; Available online 7 January 2021

\author{
Keywords: \\ cell cycle; \\ checkpoint; \\ protein kinase; \\ signaling; \\ ubiquitination
}

\begin{abstract}
Abbreviations:
CAIM, cyclin A interacting motif; CBF, cyclin-box fold;

CDK, cyclin-dependent kinase; CKI, cyclin-dependent kinase inhibitor; CKS1, cyclin-dependent kinases regulatory subunit 1 ; DSF, differential scanning

fluorimetry; GST, Glutathione-S-transferase; HDX-MS, hydrogen-deuterium exchange mass spectrometry; ITC, isothermal titration calorimetry; LRR, leucine rich repeats; RMSD, root mean square deviation; SAXS, small angle $X$-ray scattering; SEC, size exclusion chromatography; SKP, S-phase kinase associated protein; SPR, surface plasmon resonance
\end{abstract}

\section{References}

1. Zhang, H. et al, (1995). p19Skp1 and p45Skp2 are essential elements of the cyclin A-CDK2 S phase kinase. Cell, 82 (6), 915-925.

2. Bai, C. et al, (1996). SKP1 connects cell cycle regulators to the ubiquitin proteolysis machinery through a novel motif, the F-box. Cell, 86 (2), 263-274.

3. Cenciarelli, C. et al, (1999). Identification of a family of human F-box proteins. Curr. Biol., 9 (20), 1177-1179.

4. Feldman, R.M. et al, (1997). A complex of Cdc4p, Skp1p, and Cdc53p/cullin catalyzes ubiquitination of the phosphorylated CDK inhibitor Sic1p. Cell, 91 (2), 221-230.

5. Skowyra, D. et al, (1997). F-box proteins are receptors that recruit phosphorylated substrates to the SCF ubiquitinligase complex. Cell, 91 (2), 209-219.

6. Lisztwan, J. et al, (1998). Association of human CUL-1 and ubiquitin-conjugating enzyme CDC34 with the F-box protein p45(SKP2): evidence for evolutionary conservation in the subunit composition of the CDC34SCF pathway. EMBO J., 17 (2), 368-383.

7. Tsvetkov, L.M. et al, (1999). p27(Kip1) ubiquitination and degradation is regulated by the SCF(Skp2) complex through phosphorylated Thr187 in p27. Curr. Biol., 9 (12), 661-664.

8. Skaar, J.R., Pagan, J.K., Pagano, M., (2013). Mechanisms and function of substrate recruitment by F-box proteins. Nat. Rev. Mol. Cell Biol., 14 (6), 369-381.

9. Lee, E., Diehl, J., (2014). SCFs in the new millennium. Oncogene, 33 (16), 2011-2018. 
10. Zheng, N., Shabek, N., (2017). Ubiquitin Ligases: Structure, Function, and Regulation. Annu. Rev. Biochem., 86, 129-157.

11. Frescas, D., Pagano, M., (2008). Deregulated proteolysis by the F-box proteins SKP2 and beta-TrCP: tipping the scales of cancer. Nat. Rev. Cancer, 8 (6), 438-449.

12. Heo, J., Eki, R., Abbas, T., (2016). Deregulation of F-box proteins and its consequence on cancer development, progression and metastasis. Semin. Cancer Biol., 36, 33-51.

13. Spruck, C. et al, (2001). A CDK-independent function of mammalian Cks1: targeting of SCF(Skp2) to the CDK inhibitor p27Kip1. Mol. Cell, 7 (3), 639-650.

14. Ganoth, D. et al, (2001). The cell-cycle regulatory protein Cks1 is required for SCF(Skp2)-mediated ubiquitinylation of p27. Nat. Cell Biol., 3 (3), 321-324.

15. Sitry, D. et al, (2002). Three different binding sites of Cks1 are required for p27-ubiquitin ligation. J. Biol. Chem., 277 (44), 42233-42240.

16. Hao, B. et al, (2005). Structural basis of the Cks1dependent recognition of p27(Kip1) by the SCF(Skp2) ubiquitin ligase. Mol. Cell, 20 (1), 9-19.

17. Montagnoli, A. et al, (1999). Ubiquitination of p27 is regulated by Cdk-dependent phosphorylation and trimeric complex formation. Genes Dev., 13 (9), 1181-1189.

18. Bashir, T. et al, (2004). Control of the SCF(Skp2-Cks1) ubiquitin ligase by the $\mathrm{APC} / \mathrm{C}(\mathrm{Cdh} 1)$ ubiquitin ligase. Nature, 428 (6979), 190-193.

19. Wei, W. et al, (2004). Degradation of the SCF component Skp2 in cell-cycle phase G1 by the anaphase-promoting complex. Nature, 428 (6979), 194-198.

20. Ji, P. et al, (2006). Skp2 contains a novel cyclin A binding domain that directly protects cyclin A from inhibition by p27Kip1. J. Biol. Chem., 281 (33), 24058-24069.

21. Ji, P. et al, (2004). An Rb-Skp2-p27 pathway mediates acute cell cycle inhibition by $\mathrm{Rb}$ and is retained in a partialpentrance Rb mutant. Mol. Cell, 16 (1), 47-58.

22. Rodier, G. et al, (2008). Phosphorylation of Skp2 regulated by CDK2 and Cdc14B protects it from degradation by APC (Cdh1) in G1 phase. EMBO J., 27 (4), 679-691.

23. Gao, D. et al, (2009). Phosphorylation by Akt1 promotes cytoplasmic localization of Skp2 and impairs APCCdh1mediated Skp2 destruction. Nat. Cell Biol., 11 (4), 397-408.

24. Lin, H.K. et al, (2009). Phosphorylation-dependent regulation of cytosolic localization and oncogenic function of Skp2 by Akt/PKB. Nat. Cell Biol., 11 (4), 420-432.

25. Bashir, T. et al, (2010). Phosphorylation of Ser72 is dispensable for Skp2 assembly into an active SCF ubiquitin ligase and its subcellular localization. Cell Cycle, 9 (5), 971-974.

26. Cen, B. et al, (2010). Regulation of Skp2 levels by the Pim1 protein kinase. J. Biol. Chem., 285 (38), 29128-29137.

27. Inuzuka, H. et al, (2012). Acetylation-dependent regulation of Skp2 function. Cell, 150 (1), 179-193.

28. Schulman, B.A. et al, (2000). Insights into SCF ubiquitin ligases from the structure of the Skp1-Skp2 complex. Nature, 408 (6810), 381-386.

29. Morgan, D.O., (2007). The cell cycle: principles of control. In: Lawrence, E. (Ed.), Primers in Biology,. Oxford University Press.

30. Malumbres, M., (2014). Cyclin-dependent kinases. Genome Biol., 15 (6), 122.

31. Adams, P.D. et al, (1999). Retinoblastoma protein contains a C-terminal motif that targets it for phosphorylation by cyclin-cdk complexes. Mol. Cell. Biol., 19 (2), 1068-1080.
32. Russo, A.A. et al, (1996). Crystal structure of the p27Kip1 cyclin-dependent-kinase inhibitor bound to the cyclin ACdk2 complex. Nature, 382 (6589), 325-331.

33. Brown, N.R. et al, (1999). The structural basis for specificity of substrate and recruitment peptides for cyclin-dependent kinases. Nat. Cell Biol., 1 (7), 438-443.

34. Lacy, E.R. et al, (2004). p27 binds cyclin-CDK complexes through a sequential mechanism involving binding-induced protein folding. Nat. Struct. Mol. Biol., 11 (4), 358-364.

35. Tsytlonok, M. et al, (2019). Dynamic anticipation by Cdk2/ Cyclin A-bound p27 mediates signal integration in cell cycle regulation. Nature Commun., 10, 1676.

36. Takeda, D.Y., Wohlschlegel, J.A., Dutta, A., (2001). A bipartite substrate recognition motif for cyclin-dependent kinases. J. Biol. Chem., 276 (3), 1993-1997.

37. Cheng, K.Y. et al, (2006). The role of the phosphoCDK2/cyclin A recruitment site in substrate recognition. J. Biol. Chem., 281 (32), 23167-23179.

38. Bhaduri, S. et al, (2015). A docking interface in the cyclin Cln2 promotes multi-site phosphorylation of substrates and timely cell cycle entry. Curr. Biol., 25 (3), 316-325.

39. Yam, C.H. et al, (1999). Regulation of cyclin A-Cdk2 by SCF component Skp1 and F-box protein Skp2. Mol. Cell. Biol., 19 (1), 635-645.

40. Brown, N.R. et al, (2015). CDK1 structures reveal conserved and unique features of the essential cell cycle CDK. Nat. Commun., 6, 6769.

41. Huang, Y. et al, (2015). The activity and stability of the intrinsically disordered Cip/Kip protein family are regulated by non-receptor tyrosine kinases. J. Mol. Biol., 427 (2), 371-386.

42. Cornwell, O. et al, (2018). Comparing hydrogen deuterium exchange and fast photochemical oxidation of proteins: a structural characterisation of wild-type and $\Delta \mathrm{N} 6 \beta(2)$ microglobulin. J. Am. Soc. Mass Spectrom., 29 (12), 2413-2426.

43. Bassermann, F., Eichner, R., Pagano, M., (2014). The ubiquitin proteasome system - implications for cell cycle control and the targeted treatment of cancer. Biochim. Biophys. Acta, Mol. Cell. Biol. Lipids, 1843 (1), 150-162.

44. Zhu, X.H. et al, (2004). Noncatalytic requirement for cyclin A-cdk2 in p27 turnover. Mol. Cell. Biol., 24 (13), 60586066.

45. Lu, Z. et al, (2014). Skp2 suppresses apoptosis in Rb1deficient tumours by limiting E2F1 activity. Nat. Commun., 5, 3463.

46. Schulze-Gahmen, U. et al, (2013). The AFF4 scaffold binds human P-TEFb adjacent to HIV Tat. Elife, 2, e00327.

47. Gu, J. et al, (2014). Crystal structure of HIV-1 Tat complexed with human P-TEFb and AFF4. Cell Cycle, 13 (11), 1788-1797.

48. Hallett, S. et al, (2017). Differential regulation of G1 CDK complexes by the Hsp90-Cdc37 chaperone system. Cell Rep., 21 (5), 1386-1398.

49. Cryar, A., Groves, K., Quaglia, M., (2017). Online hydrogen-deuterium exchange traveling wave ion mobility mass spectrometry (HDX-IM-MS): a systematic evaluation. J. Am. Soc. Mass Spectrom., 28 (6), 1192-1202.

50. Rambo, R.P., Tainer, J.A., (2015). Modeling macromolecular motions by $\mathrm{x}$-ray-scattering-constrained molecular dynamics. Biophys. J., 108 (10), 2421-2423.

51. Franke, D., Svergun, D.I., (2009). DAMMIF, a program for rapid ab-initio shape determination in small-angle scattering. J. Appl. Crystallogr., 42 (Pt 2), 342-346. 
52. Petoukhov, M.V. et al, (2012). New developments in the ATSAS program package for small-angle scattering data analysis. J. Appl. Crystallogr., 45 (Pt 2), 342-350.

53. Goddard, T.D. et al, (2018). UCSF ChimeraX: Meeting modern challenges in visualization and analysis. Protein Sci., 27 (1), 14-25.

54. Potterton, L. et al, (2018). CCP4i2: the new graphical user interface to the CCP4 program suite. Acta. Crystallogr. Sect. D: Struct. Biol., 74 (Pt 2), 68-84.

55. Emsley, P. et al, (2010). Features and development of Coot. Acta Crystallogr. D Biol. Crystallogr., 66 (Pt 4), 486501.

56. McNicholas, S. et al, (2011). Presenting your structures: the CCP4MG molecular-graphics software. Acta. Crystallogr. Sect. D, 67 (Pt 4), 386-394.
57. Marzio, A. et al, (2019). The F-box domain-dependent activity of EMI1 regulates PARPi sensitivity in triplenegative breast cancers. Mol. Cell, 73 (2), 224-237.e6.

58. Honda, R. et al, (2005). The structure of cyclin E1/CDK2: implications for CDK2 activation and CDK2-independent roles. EMBO J., 24 (3), 452-463.

59. Perry, J.J., Tainer, J.A., (2013). Developing advanced Xray scattering methods combined with crystallography and computation. Methods, 59 (3), 363-371.

60. Webb, B., Sali, A., (2021). Protein Structure Modelling with MODELLER. Methods Mol. Biol., 2199, 239-255.

61. Zimmermann, L. et al, (2018). A completely reimplemented MPI bioinformatics toolkit with a new HHpred server at its core. J. Mol. Biol., 430, 2237-2243. 